\title{
Suppressive Lateral Interactions at Parafoveal Representations in Primary Visual Cortex
}

\author{
Arezoo Pooresmaeili, ${ }^{1 *}$ Jose L. Herrero, ${ }^{2 *}$ Matthew W. Self, ${ }^{1}$ Pieter R. Roelfsema, ${ }^{1,3}$ and Alexander Thiele ${ }^{2}$ \\ ${ }^{1}$ Department of Vision and Cognition, The Netherlands Institute for Neuroscience, An Institute of the Royal Netherlands Academy of Arts and Sciences, \\ 1105 BA Amsterdam, The Netherlands, ${ }^{2}$ Institute of Neuroscience, University of Newcastle upon Tyne, Newcastle upon Tyne NE2 4HH, United Kingdom, \\ and ${ }^{3}$ Department of Integrative Neurophysiology, Centre for Neurogenomics and Cognitive Research, Vrije Universiteit, 1081 HV Amsterdam, \\ The Netherlands
}

The perceptual salience and visibility of image elements is influenced by other elements in their vicinity. The perceptual effect of image elements on an adjacent target element depends on their relative orientation. Collinear flanking elements usually improve sensitivity for the target element, whereas orthogonal elements have a weaker effect. It is believed that the collinear flankers exert these effects through lateral interactions between neurons in the primary visual cortex (area V1), but the precise mechanisms underlying these contextual interactions remain unknown. Here, we directly examined this question by recording the effects of flankers on the responses of $\mathrm{V} 1$ neurons at parafoveal representations while monkeys performed a fixation task or a contrast detection task. We found, unexpectedly, that collinear flankers reduce the monkeys' perceptual sensitivity for a central target element. This behavioral effect was explained by a flanker-induced increase in the activity of V1 neurons in the absence of the central target stimulus, which reduced the amplitude of the target response. Our results indicate that the dominant effect of collinear flankers in parafoveal vision is suppression and suggest that these suppressive effects are caused by a decrease in the dynamic range of neurons coding the central target.

\section{Introduction}

The spatial context embedding an image element has a strong influence on the perception of the image element itself (Gilbert, 1998; Albright and Stoner, 2002). This contextual effect is often explained by lateral interactions between neurons representing the relevant image element and adjacent flanking elements that can either facilitate or suppress its detection (for review, see Seriès et al., 2003).

In psychophysics, facilitation has been observed for foveally presented, low-contrast Gabor targets flanked by collinear Gabor elements. This effect is strongest when the distance of the flankers is two to four times the wavelength $(\lambda)$ of the Gabor element (Polat and Sagi, 1993, 1994; Morgan and Dresp, 1995). In contrast, suppression occurs for flankers located closer to the target $(<2 \lambda)$, especially if they are not collinear, and/or the target stimulus has a high contrast (Adini and Sagi, 2001; Chen and Tyler, 2002). Facilitatory flanker effects occur at the fovea, but they are less consistent at more eccentric representations (Williams and Hess, 1998; Macknik et al., 2000; Zenger-Landolt and Koch, 2001; Shani and Sagi, 2005; Macknik, 2006).

Received Dec. 8, 2009; revised May 20, 2010; accepted May 28, 2010.

The work in Amsterdam was supported by a Netherlands Organization for Scientific Research-Vici grant and a Human Frontier Science Program grant. The work in Newcastle was supported by the Biotechnology and Biological Sciences Research Council, the Wellcome Trust, and the Medical Research Council, and by the excellent technical support by the Comparative Biology Centre. We thank Kor Brandsma and Dave Vleesenbeek for excellent technical support.

*A.P. and J.L.H. contributed equally to this work.

Correspondence should be addressed to Pieter R. Roelfsema, The Netherlands Institute for Neuroscience, Meibergdreef 47, 1105 BA Amsterdam, The Netherlands. E-mail: p.roelfsema@nin.knaw.nl.

DOI:10.1523/JNEUROSCI.6071-09.2010

Copyright $\odot 2010$ the authors $\quad 0270-6474 / 10 / 3012745-14 \$ 15.00 / 0$
The neuronal mechanisms underlying these lateral interactions may reside at the level of the primary visual cortex (Kapadia et al., 1995). Neurophysiological studies have shown that collinear flankers increase V1 neuronal responses to a central target (Mizobe et al., 2001; Crook et al., 2002), especially if the target contrast is low (Polat et al., 1998; Kapadia et al., 2000). Orthogonal flankers, however, exert a weaker facilitative effect or even suppress the neuronal activity (Kapadia et al., 1995; Das and Gilbert, 1999).

The increase in neuronal activity evoked by a central target in the presence of collinear flankers may therefore account for the improved contrast sensitivity. However, the percentage of neurons in the primary visual cortex supporting the psychophysical results is relatively small. For example, Chen et al. (2001) found that only $38 \%$ of the neurons recorded showed facilitation at low contrast and suppression at high contrast, whereas the rest of the neurons showed either suppressive or opposite effects. Even lower percentages were observed by others [34\% by Polat et al. (1998); 14\% by Ito and Gilbert (1999)]. Controversially, most of the neurophysiological studies reporting flanker facilitation were executed at more eccentric representations, where the perceptual effects of flankers are mostly suppressive. In addition, interpretation of flanker-related effects from anesthetized or awake-nonbehaving animals is not easy, as anesthesia (Lamme et al., 1998) and attentional states (Freeman et al., 2001; Roberts et al., 2007) have been shown to influence the strength of contextual effects.

Here, we try to clarify the discrepancy between the psychophysical and neurophysiological literature at parafoveal representations. First, in alert fixating macaques, we recorded contrast 
response functions (CRFs) of V1 neurons in the absence and presence of collinear flankers at various distances $(\sim 1,2$, or $3 \lambda)$. Neuronal thresholds should be reduced if flankers are to facilitate contrast detection (i.e., at $3 \lambda$ ), and increased otherwise (i.e., at $<3 \lambda$ ). In a second set of experiments, we compared neuronal and perceptual thresholds by recording CRFs of V1 neurons while animals engaged in a contrast detection task. In line with recent reports using optical and voltage-sensitive dye imaging (Kinoshita et al., 2009; Meirovithz et al., 2010), we find that collinear flankers suppress neuronal responses evoked by the central target at all flanker distances and increase ongoing activity at the closer ones. These effects are associated with a decrease in contrast sensitivity at the behavioral level.

\section{Materials and Methods}

The experiments were performed in two laboratories. The first, singleunit experiment was performed in Newcastle, and the experiment in which behavioral contrast sensitivity was measured in combination with multiunit recording, in Amsterdam. We will first describe the methods for single-unit recordings and then describe the methods for the multiunit recordings in combination with the measurement of contrast detection performance.

All experiments were performed in accordance with the European Communities Council Directive 1986 (86/609/EEC) and the National Institutes of Health Guidelines for the Care and Use of Animals for Experimental Procedures. Additionally, the experiments in Newcastle were performed in accordance with the United Kingdom Animals Scientific Procedures Act, whereas the experiments in Amsterdam were approved by the Institutional Animal Care and Use Committee of the Royal Netherlands Academy of Arts and Sciences.

\section{Single-cell recordings during visual fixation (Newcastle data)}

We recorded neurons in two male adult rhesus monkeys (Macaca mulatta). After initial training, monkeys were implanted with a head holder, eye coil, and recording chambers above V1 under general anesthesia and sterile conditions. All details of surgical procedures, postoperative care, and the cleaning of the implant and recording chambers have been published previously (Thiele et al., 2006).

Electrophysiological recordings and behavioral procedures. Single-cell discharges were recorded extracellularly using tungsten-in-glass microelectrodes (0.5-2.5 M $\Omega$, made in-house). Stimulus presentation and behavioral control was managed by Remote Cortex 5.95 (Laboratory of Neuropsychology, National Institute for Mental Health, Bethesda, MD). Neuronal data were collected by Cheetah data acquisition (Neuralynx; 30 $\mathrm{kHz}$ sampling rate) interlinked with Remote Cortex 5.95.

Monkeys were trained to keep fixation (eye window, $1.2^{\circ}$ in diameter) while a small oriented Gabor was presented in the periphery of their visual field, with or without two collinear flankers (see Fig. $1 A$ ). The fixation point (FP) $\left(0.1^{\circ}\right.$ diameter) was presented centrally against a gray background $\left(21 \mathrm{~cd} / \mathrm{m}^{2}\right)$ on a 20 inch analog cathode ray tube (CRT) monitor $(100 \mathrm{~Hz} ; 1600 \times 1200$ pixels; $57 \mathrm{~cm}$ from the animal). Eye position was recorded with an infrared-based camera system (Thomas Recording) and sampled at a rate of $250 \mathrm{~Hz}$.

A trial started as soon as the monkey's eye position was within a fixation window centered on the fixation point. After $500 \mathrm{~ms}$, a set of four oriented Gabor stimuli was presented for $700 \mathrm{~ms}$ each with $300 \mathrm{~ms}$ gaps between presentations. At the end of the four presentations, the fixation point disappeared, and monkeys were rewarded if their eye position had been within the fixation window for the trial duration. If the monkey broke fixation before the FP disappeared, the condition was repeated later in the block. Twenty trials per stimulus and contrast condition were recorded in most recordings. Cells were excluded if $<10$ trials per stimulus and contrast were available.

Stimuli. Stimuli consisted of either central Gabor elements presented in isolation or flanked by two isooriented flankers. The orientation and spatial frequency of the Gabors matched the preference of the neuron (see below). Each Gabor moved within a Gaussian aperture at $4 \mathrm{~Hz}$ temporal frequency. The motion was perpendicular to the orientation of the Gabor, and reversed direction at a frequency of $4 \mathrm{~Hz}$. Within the sequence of four presentations per trial, the order of stimulus presentation within a trial and between trials was randomized.

Central and flanker Gabors were identical in all respects except for their contrasts. The contrast of the central Gabor was varied between 0,4 , $8,12,16,24,32$, and $64 \%$ (Michelson contrast), whereas the contrast of the flanking Gabors was fixed at $48 \%$. The distance between the central and flanking Gabors could be one, two, or three to four times the Gabor wavelength $(\lambda)$. The exact distance varied slightly with the spatial frequency preference. High spatial frequencies of, for example, $6 \mathrm{cyc} /{ }^{\circ}$ would result in center-flanker distances of $0.166^{\circ}$ at a distance of $1 \lambda$, whereby the receptive field (RF) center would be filled by flankers. To account for this, we used distances of $1.5,2.8$, and $4 \lambda$ for spatial frequencies of $\geq 6 \mathrm{cyc} /{ }^{\circ}$, and distances of 1,2 , and $3 \lambda$ for spatial frequencies of $<6$ $\mathrm{cyc} /{ }^{\circ}$. The large majority of our neurons preferred spatial frequencies of $\leq 4 \mathrm{cyc} /{ }^{\circ}$, and only eight neurons were measured with $\geq 6 \mathrm{cyc} /{ }^{\circ}$. We included them in our overall sample and treated them as if the wavelengths had been 1,2 , and $3 \lambda$, respectively. We also scaled the size of our stimuli, whereby the half-width at half-height of the Gaussian envelope was 0.3 times the spatial frequency for spatial frequencies of $<2 \mathrm{cyc} /{ }^{\circ}$, it was 0.4 times the spatial frequency for spatial frequencies between 2 and $4 \mathrm{cyc} /{ }^{\circ}, 0.5$ times the spatial frequency for spatial frequencies between 4 and $6 \mathrm{cyc} /{ }^{\circ}$, and 0.6 times the spatial frequency for spatial frequencies of $\geq 6 \mathrm{cyc} /{ }^{\circ}$.

Receptive field characterization. Receptive fields were mapped by presenting a $0.1^{\circ}$ black $(100 \%$ contrast) square at pseudorandom locations on a $10 \times 10 \operatorname{grid}\left(\mathrm{a} 1 \times 1^{\circ}\right.$ area; five repetitions at each location; $100 \mathrm{~ms}$ presentation time with $100 \mathrm{~ms}$ gaps) while monkeys fixated centrally on the CRT monitor. The mean response at each stimulus location (calculated from 30 to $100 \mathrm{~ms}$ after stimulus onset) was determined, and a two-dimensional Gaussian was fitted to the response distribution. The RF center was taken as the location of the peak of the fitted Gaussian (Roberts et al., 2007). The mean receptive field eccentricity was $3.25^{\circ}$ in monkey $\mathrm{D}$ and $5.6^{\circ}$ in monkey $\mathrm{H}$.

Optimal spatial frequency (in conjunction with orientation and phase) was determined by a reversed correlation technique (DeAngelis et al., 1994). Monkeys fixated centrally on the CRT monitor while 336 circular patches of static sinusoidal gratings $\left(1^{\circ}\right.$ diameter $)$ were presented for $60 \mathrm{~ms}$ in a pseudorandomized order. The patches were centered over the minimum response field and varied in orientation (12 orientations, $\left.0-165^{\circ}\right)$, spatial frequency $\left(1,3,5,7,8,9,10 \mathrm{cyc}^{\circ}\right)$, and phase $(0,0.5 \pi$, $1 \pi, 1.5 \pi)$. Responses were averaged in a time window from 30 to $90 \mathrm{~ms}$ (60-120 ms for neurons with a late-onset response) with 5-10 repetitions per stimulus. The stimulus that yielded the peak response was taken to represent the preferred orientation, spatial frequency, and phase of the neuron under study. The obtained parameters were used to determine the spatial frequency and orientation of the central and the flanking Gabors, which had identical properties.

Analysis of the physiological data. In a total of 72 cells from two monkeys, we tested whether the contrast of the central Gabor or the presence of flankers significantly affected neuronal activity and whether there was a significant interaction between these factors. We used the response period from 200 to $700 \mathrm{~ms}$ after stimulus onset for our analysis. Neurons were analyzed further if contrast and flanker presence significantly affected firing rates, or if a significant interaction between contrast and flanker occurred (ANOVA, $p<0.05$ ). A total of 67 of 72 cells ( 48 from monkey D; 19 from monkey $\mathrm{H}$ ) passed the basic statistical test (twofactor ANOVA: factor 1, contrast; factor 2, flanker presence). For the initial recordings, we used two target-flankers spatial distances ( 1 and $3 \lambda$ ) instead of three ( 31 of 67 cells; all recorded in monkey D), but in our later recordings, we used three flanker distances to increase the flanker distance sampling density.

We quantified the target-evoked response for each contrast level $c$ at all measured target-flanker separation distances. This was done by subtracting neuronal responses elicited in a period 200-700 ms after stimulus onset by the presence of flankers alone $\left(R_{\text {flankers }}\right)$ from the responses when the flankers were presented in conjunction with the target, $R_{\text {target }}(c)=R_{\text {target }+ \text { flankers }}(c)-R_{\text {flankers }}$.

To determine the effect of the flankers on contrast tuning, contrast response functions were obtained for each neuron. Each contrast re- 
sponse function was based on the $R_{\text {target }}(c)$ to $10-30$ repetitions of each contrast and a total of 80-160 stimulus repetitions for each flanker condition. Contrast response functions were fitted for each flanker condition with a hyperbolic ratio function of the following form:

$$
R_{\text {target }}(c)=R_{\text {target_max }} *\left(c^{n} /\left[c^{n}+c_{50}^{n}\right]\right)+M,
$$

where $R_{\text {target_max }}$ is the saturated response, $c_{50}$ is the contrast at which the half-maximal response is reached, $n$ determines the slope of the contrast response function, and $M$ corresponds to the spontaneous activity. This model provides a good approximation of contrast response functions in monkey visual cortex (Albrecht and Hamilton, 1982; Thiele et al., 2004; Williford and Maunsell, 2006), and we used multidimensional unconstrained nonlinear minimization (Nelder-Mead) to minimize the summed squared difference between data and model (Matlab 7.1; Mathworks). To determine whether $R_{\text {target_max }}$ or $c_{50}$ differed significantly when the flankers were introduced, we fitted each function independently with the hyperbolic ratio function and determined the $\chi^{2}$ error of the individual fits, and also when fits were forced to obtain the same $R_{\text {target_max }}\left(c_{50}\right)$. The difference of the $\chi^{2}$ errors for the two approaches can be used to test whether the parameter of interest significantly changes when flankers are presented (Watson, 1979).

\section{Multiunit recording in the contrast detection task (Amsterdam)}

Behavioral task. We used two macaque monkeys for our electrophysiological and behavioral experiments. The monkeys were trained to detect a small oriented Gabor target presented with or without two collinear flankers (contrast detection task depicted in Fig. 5). The monkeys were trained on this task for 1-2 months until their performance stabilized. The stimuli were presented on a CRT color monitor (21 inch with a resolution of $1024 \times 768$ and frame rate of $75 \mathrm{~Hz}$ ) calibrated (gamma correction) by using a 8 bit RGB lookup table. The stimuli were viewed binocularly from a distance of $75 \mathrm{~cm}$ from the monitor. The eye position was monitored with the double induction technique (Bour et al., 1984) and sampled at a rate of $900 \mathrm{~Hz}$. At the beginning of each trial, a black fixation point $\left(0.2^{\circ}\right.$ in monkey $\mathrm{G}$ and $0.4^{\circ}$ in monkey $\left.\mathrm{A}\right)$ appeared in the central position against a homogenous gray background (luminance, $16.3 \mathrm{~cd} / \mathrm{cm}^{2}$ ). In trials in which flankers were presented, two horizontal Gabor elements appeared on the screen at the same time as the fixation point. A trial started as soon as the monkey's eye position was within a $1.1^{\circ} \times 1.1^{\circ}$ window centered on the fixation point. An initial fixation interval of $500 \mathrm{~ms}$ was followed by a stimulus presentation epoch. During this epoch, in one-half of the trials (target-present trials) a Gabor stimulus of varying contrasts was displayed, whereas in the other one-half of the trials the target was absent. The stimulus presentation epoch lasted for $200 \mathrm{~ms}$ and was followed by a second fixation interval of $400 \mathrm{~ms}$. At the end of this interval, the fixation point disappeared and the monkeys were required to respond by either making an eye movement to a black circle (size, $0.6^{\circ}$ ) displayed at the location at which the Gabor target had been presented if they had seen the target or by maintaining gaze within the fixation window for $500 \mathrm{~ms}$ if they had not seen the target. Correct responses were rewarded by a drop of apple juice.

Target-present trials with the various contrasts (in total, $50 \%$ of trials) and target-absent trials (the other 50\%) were randomly interleaved. Trials with and without collinear flankers were presented in separate blocks, with blocks alternating between the two conditions. This was done to avoid the possibility that the monkeys used a similar response criterion, as mixed stimuli have been shown to undermine accuracy (Gorea et al., 2005). Trials with the three different distances between target and flankers were run on different days with 10-25 blocks of trials per day, each consisting of 60-100 trials. Across all the recording sessions, we collected at least 70 trials for each stimulus condition. To derive the population data (see Fig. $7 B-D$ ), we pooled across different recording sessions in which the same stimulus configuration had been used.

We investigated the effect of flanker orientation in separate sessions in which the monkeys performed a passive fixation task (data presented in Fig. 7A). In this experiment, the Gabor targets and the central target were presented at the same time, after $500 \mathrm{~ms}$ of steady fixation (target and flankers appeared at different times in the main experiment). The stimuli disappeared after $200 \mathrm{~ms}$, and the monkeys had to maintain fixation for another $400 \mathrm{~ms}$.

Stimuli. Gabor stimuli were Gaussian-windowed, sinusoidal luminance gratings (carrier wavelength $\lambda=0.44^{\circ}$; Gaussian $\sigma=0.2^{\circ}$ in monkey $\mathrm{G}$ and $0.3^{\circ}$ in monkey $\mathrm{A}$; and carrier spatial frequency, $2.3 \mathrm{cyc} /^{\circ}$ ) added to a uniform background. We used six center contrasts, $0,3,4,6$, 9,14 , and $20 \%$ (Michelson contrast), in monkey G and added a seventh contrast of $60 \%$ in monkey A. All contrast levels were presented with equal probability. The flanker contrast was fixed at a value of $13 \%$. The stimuli were placed at the center of the receptive fields, at an eccentricity of $1.5^{\circ}$ in monkey $\mathrm{G}$ and at $2.5^{\circ}$ in monkey A. The horizontal axis of the Gaussian envelop was scaled by a factor of 1.5 relative to the vertical axis, as this transformation has been shown to increase the facilitatory effects of flankers on contrast perception in humans (Polat and Tyler, 1999). The orientation of the Gabors was $0^{\circ}$ (vertical) or $90^{\circ}$ (horizontal). Target and flanker Gabors were identical in all respects except for their contrasts. The center-to-center distance between target and flankers was 2.8, 3.5 , or $4.2 \lambda$ (corresponding to $1.25,1.55$, and 1.85 degrees of visual angle, respectively).

Recording of multiunit activity in area V1. In a first operation, a head holder was implanted and a gold ring was inserted under the conjunctiva of one eye for the measurement of eye position. In a second operation, arrays of $4 \times 5$ or $5 \times 5$ electrodes (Cyberkinetics Neurotechnology Systems) were chronically implanted in area V1. The operations were performed under aseptic conditions and general anesthesia. Details of the surgical procedures and the postoperative care have been described previously (Roelfsema et al., 1998). Extracellular activity was recorded with TDT (Tucker-Davis Technologies) multichannel recording equipment. For the detection of multiunit activity (MUA), the signal was amplified, bandpass filtered (300-9000 Hz), full-wave rectified, low-pass filtered $(<200 \mathrm{~Hz})$, and sampled at a rate of $760 \mathrm{~Hz}$. The MUA provides an instantaneous measure of the number and the size of action potentials of neurons in the vicinity of the electrode tip (Supèr and Roelfsema, 2005).

When the monkeys had fully recovered from the operation, we first mapped the dimensions of the receptive fields by measuring the onset and offset times of the visual response to a slowly moving light bar, for each of the eight movement directions (Supèr and Roelfsema, 2005). In both monkeys the receptive fields were located at the lower left visual field. The median area of the receptive fields was $0.76 \mathrm{deg}^{2}$ (range from 0.12 to $2.03 \mathrm{deg}^{2}$ ). The median receptive field eccentricity was $2.5^{\circ}$ in monkey A (range from 2.1 to $2.83^{\circ}$ ) and $1^{\circ}$ in monkey G (range from 0.87 to $1.87^{\circ}$ ). We determined the orientation tuning of the recording sites by using the responses to the moving bar stimulus. The responses were averaged in a time window extending from the onset to the offset of the bar. The responses to the bars that had the same orientation but moved in different directions were averaged together. The preferred orientation of a recording site was determined as the orientation that induced the maximum response.

Analysis of the behavioral data. As a measure of detection sensitivity, we computed $d^{\prime}$ as follows: $d^{\prime}=Z\left(P_{\text {hit }}\right)-Z\left(P_{\mathrm{FA}}\right)$, where $Z\left(P_{\text {hit }}\right)$ denotes the $Z$-transform of the probability of hit and $Z\left(P_{\mathrm{FA}}\right)$ denotes the $Z$-transform of the probability of false alarm. Psychometric curves (see Fig. $6 B$ ) were generated by computing the $d^{\prime}$ values for the various contrast levels and were fitted by a Weibull function $F(c)$ as follows:

$$
F(c)=\delta\left[1-e^{-(c / \alpha)^{\beta}}\right] .
$$

Here, $c$ is the contrast level, $F(c)$ is the estimated $d^{\prime}$ for each contrast level, and the three free parameters $\delta, \alpha$, and $\beta$ correspond to the asymptote, offset, and slope of the function, respectively. The contrast thresholds were defined as the contrast level $c$, where $F(c)$ reaches a $d^{\prime}$ value of 1.5 (i.e., a correct rate of $\sim 70 \%$ if the false alarm is $\sim 15 \%$ ). We used a multidimensional unconstrained nonlinear minimization (NelderMead) algorithm for minimizing the summed squared difference between data and model (Matlab 7.1; Mathworks).

To assess whether flankers significantly affected the behavioral performance, for each measure of behavior (probability of false alarm, $d^{\prime}$, contrast threshold), we generated a bootstrap distribution by resampling the data with replacement $(N=1000)$. This procedure was performed for 
the trials with and without flankers and a distribution of differences between them was computed to determine statistical significance (twosided test). An effect was considered significant if the value of $p<0.05$.

Analysis of the neuronal activity. Peristimulus time histograms (PSTHs) were calculated in a time window from $500 \mathrm{~ms}$ before stimulus onset to $600 \mathrm{~ms}$ thereafter, and were normalized to the peak response (Pe) after subtraction of the spontaneous activity (Sp). The spontaneous activity (Sp) was computed as the average activity in the $500 \mathrm{~ms}$ fixation interval before the stimulus onset in the absence of flankers. The peak response $(\mathrm{Pe})$ was determined as the maximum evoked response by the central target (center-only condition) with a contrast of $20 \%$ in a time window from 50 to $150 \mathrm{~ms}$ after the stimulus onset. The average PSTHs and the single trial responses were normalized by first subtracting Sp and dividing the result by the peak response (Pe-Sp). We included a recording site in our analysis if the ratio of the maximum stimulus-evoked response (Pe-Sp) to the $\mathrm{SD}$ of the spontaneous activity, $\sigma_{\mathrm{Sp}}$, reached a criterion value $(>1)$. Contrast response functions (see Fig. 8) were generated by subtracting the responses on target-absent trials from the responses on target-present trials (averaged in a window from 50 to $350 \mathrm{~ms}$ after the stimulus onset). The $R_{\text {target_max }}$ and $c_{50}$ values were estimated by fitting a hyperbolic ratio function to the contrast response function of each recording site, similar to the procedure used for the single-cell data.

We computed the neural $d^{\prime}$ as follows:

$$
\begin{gathered}
d^{\prime}=\frac{\left(\mu_{2}-\mu_{1}\right)}{\sigma}, \text { with } \\
\sigma=\sqrt{\frac{\left(n_{1}-1\right) \sigma_{1}^{2}+\left(n_{2}-1\right) \sigma_{2}^{2}}{n_{1}+n_{2}-2} .}
\end{gathered}
$$

$\mu_{2}$ and $\mu_{1}$ represent the mean activity, and $\sigma_{2}$ and $\sigma_{1}$ denote the SD of the activity in target-present and target-absent trials, respectively.

Measurement of the latency of the neuronal responses. We estimated the latency of responses by fitting a function $f(t)$ to the single-unit (see Fig. $2 A$ ) or multiunit (see Fig. 7A) responses (Roelfsema et al., 2003, 2007). The shape of $f(t)$ was derived from the following two assumptions: (1) the onset of the response has a Gaussian distribution across trials and across neurons, and (2) a fraction of the response dissipates exponentially. These assumptions yield the following two differential equations: $\partial m_{1}(t) /$ $\partial t=-\alpha m_{1}(t)+g(t, \mu, \sigma)$ for the dissipating response, and $\partial m_{2}(t) / \partial t=$ $g(t, \mu, \sigma)$ for the nondissipating response. Here, $m_{1}(t)+m_{2}(t)=f(t)$ is the total response, $g(t, \mu, \sigma)$ is a Gaussian density with mean $\mu$ and $\mathrm{SD} \sigma$, and $\alpha^{-1}$ is the time constant of dissipation. The solution to these equations is the sum of an ex-Gaussian and a cumulative Gaussian, which was fitted to the response as follows:

$$
f(t)=d \cdot \operatorname{Exp}\left(\mu \alpha+0.5 \sigma^{2} \alpha^{2}-\alpha t\right) \cdot G\left(t, \mu+\sigma^{2} \alpha, \sigma\right)+c \cdot G(t, \mu, \sigma) .
$$

Thus, $f(t)$ is determined by five parameters, $\mu, \sigma, \alpha, c$, and $d ; G(t, \mu, \sigma)$ is a cumulative Gaussian, and $c$ and $d$ are the contributions of nondissipating and dissipating response, respectively. The latency of the visual response (lat $\left.{ }_{\text {onset }}\right)$ was (arbitrarily) defined as the point in time that the fitted function reached $33 \%$ of its maximum (lat 33 ). To compute a $95 \%$ confidence interval for the latency of the visual response, we used a bootstrapping procedure. If there are $N$ recording sites, we randomly selected $N$ cases with replacement and determined the latency in the simulated sample using the curve-fitting method described above. We repeated this procedure 10,000 times to estimate the $95 \%$ confidence interval.

\section{Results}

We will first describe the results of the single-unit recording experiments in area $\mathrm{V} 1$ and then continue with the results obtained with V1 multiunit recording during a contrast detection task.

\section{Effects of collinear flankers on single-unit activity of V1 neurons}

In our first experiment, monkeys were engaged in a fixation task while they passively viewed a sequence of Gabor elements that were presented in the receptive field of a single neuron in area $\mathrm{V} 1$ (Fig. 1A). Figure $1 B$ illustrates the PSTHs of an example neuron to the central target stimulus at eight contrast levels when it was presented alone or together with two collinear flankers. In the absence of the flankers and without central stimulation of the receptive field, the firing rate of the neuron remained at the level of the spontaneous activity. The appearance of a central Gabor then elicited a response that increased with the contrast of the stimulus. When two collinear flankers were presented without the central target, the responses of the neuron also increased above the spontaneous activity level. This response was significant for close flankers $(1$ and $2 \lambda)(p<0.001$ and $p<0.02$, respectively; signed rank test). The responses to the far flankers $(3 \lambda)$ were not significantly different from the spontaneous activity of the neuron ( $p>0.3$, signed rank test). To determine the amount of activity evoked by the central target in the presence of flankers (Resp target $)$, we subtracted the responses to the flankersonly condition ( Resp $_{\text {flankers }}$ ) from responses to the central target accompanied by the flankers (Resp $p_{\text {target }+ \text { flankers }}$ ). The shaded gray area below the $x$-axis of Figure $1 B$ depicts Resp target $_{\text {for the stim- }}$ ulus of $8 \%$ contrast. Compared with the center-only condition, the presence of the nearest flankers (at 1 and $2 \lambda$ ) reduced responses to the central target. We constructed contrast response functions of neurons by computing the average Resp target $_{\text {in a }}$ time window from 200 to $700 \mathrm{~ms}$ after the stimulus onset for every contrast level (Fig. 1C). Flanker-induced facilitation/inhibition was quantified by fitting a hyperbolic ratio function to the contrast response function of the neurons (see Materials and Methods). We anticipated two possible neuronal correlates of the flanker effect. First, the flankers might increase or decrease the response maximum $\left(R_{\text {target_max }}\right)$. Second, the flankers could increase or decrease the contrast at which the half-maximal response is obtained $\left(c_{50}\right)$. An increase in $R_{\text {target_max }}$ or a decrease in $c_{50}$ would indicate flanker facilitation and the reverse would indicate flanker suppression. In the example cell in Figure 1C, the flankers caused a reduction in the $R_{\text {target_max }}$ at all target contrast levels. Fitted $R_{\text {target_max }}$ was reduced from 24 spikes/s in center-only condition to $6.6,18$, and 23 spikes/s in the presence of flankers at 1,2 , and $3 \lambda$, respectively. $R_{\text {target_max }}$ was significantly reduced for all three flanker distances $(p<$ 0.001 ). Moreover, the flankers significantly increased $c_{50}$ for all three flanker distances $(p<0.001)$. The value of $c_{50}$ increased from a value of $10.2 \%$ in the absence of flankers to $17.8,22.2$, and $16.2 \%$ in the presence of flankers at 1,2 , and $3 \lambda$, respectively.

Figure $1 D$ shows the contrast response function of another neuron. The cell was strongly suppressed by flankers at a distance of $1 \lambda$, but we observed a facilitatory effect of the far flankers $(2$ and $3 \lambda$ ) as reflected by an increase in $R_{\text {target_max }}$ from 30 spikes/s in the absence of flankers to 34 and 36 spikes/s for flankers at 2 and $3 \lambda$, respectively ( $p<0.01, \chi^{2}$ fitting) (see above). Flankers had variable effects on $c_{50}$ for this neuron; it changed from $10.4 \%$ in the absence of flankers to $16.4 \%$ for a flanker distance of $2 \lambda$ $(p<0.001)$ and to $11.3 \%$ for a flanker distance of $3 \lambda(p=$ 0.082 ). Please note that the facilitation at larger flanker distances that was observed in this neuron was exceptional in our sample. In most of the cells, flankers reduced responses to center targets at all distances tested (see below). Thus, the contrast response functions of the cell shown in Figure $1 C$ are more representative of the effects observed at the population level.

As shown for our example neurons, nearby collinear flankers often induced responses in the absence of the central target. The degree of flanker-induced activation depended on flanker dis- 
A

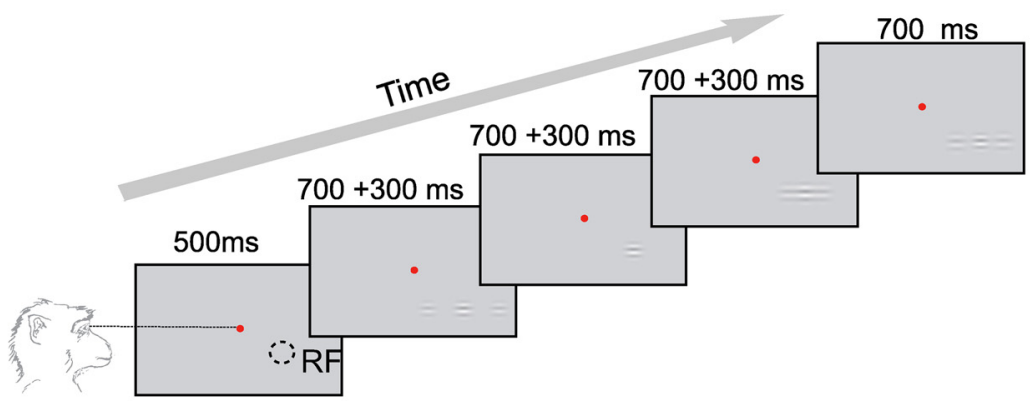

B
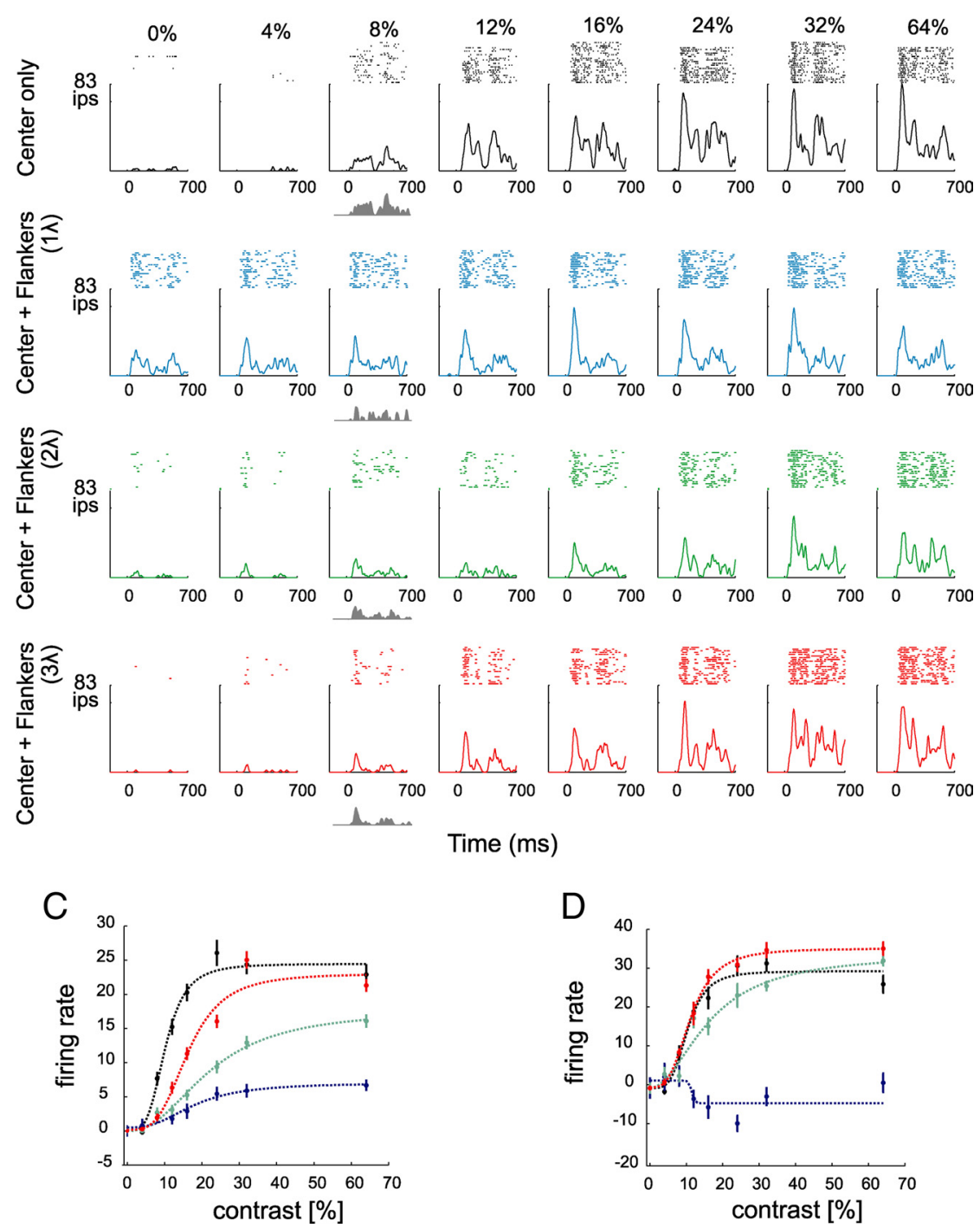

Figure 1. Effects of collinear flankers on the activity of V1 neurons. A, The fixation task. The monkeys fixated a central fixation point (red) and passively viewed a sequence of four stimuli. Each stimulus consisted of a central target Gabor that was presented either in isolation or together with two collinear flankers. Stimulus presentation was $700 \mathrm{~ms}$ with an interstimulus interval of $300 \mathrm{~ms}$. RF denotes receptive field location. $\boldsymbol{B}$, Activity of a $\mathrm{V} 1$ neuron evoked by the presentation of the center stimulus alone or in the presence of collinear flankers located at one of three distances (1,2, and $3 \lambda)$. The gray shaded area represents the net response evoked by a target with a contrast of $8 \%$. C, Contrast response function of the neuron shown in $\boldsymbol{B}$. Note that the cell shows suppression at all contrast levels and every flanker distance, as was typical in our sample. The curves show fits of a hyperbolic ratio function. $\boldsymbol{D}$, Contrast response function of a different, more exceptional neuron that showed some degree of facilitation for high-contrast stimuli in the presence of far ( 2 and $3 \lambda)$ flankers. Error bars indicate SEM. an absolute distance from the center of the target to the center of the flanker of $0.25^{\circ}$ for a spatial frequency of $4 \mathrm{cyc} /{ }^{\circ}$, and the flankers therefore infringe on the CRF of the neurons, whereas one wavelength corresponds to a distance of $1^{\circ}$ for a spatial frequency of $1 \mathrm{cyc} /{ }^{\circ}$, which may not infringe on the CRF if it is small (e.g., 0.5 diameter). We characterized this flankersinduced activity (Resp flankers $_{\text {) across the }}$ population of V1 neurons (Fig. 2A). We computed the magnitude and the latency of responses to the collinear flankers when they were presented without the central target and compared these responses to the activity evoked by the target. A target placed inside the CRF of the neurons (contrast 32\%) caused a strong response with a latency of $80 \mathrm{~ms}$ ( $95 \%$ confidence interval, 76-84 ms). Flankers at 1 and $2 \lambda$ presented in isolation (no center stimulus) elicited responses that were significantly higher than the baseline activity (both values of $p<10^{-5}$, Mann-Whitney $U$ test) but weaker (both values of $p<$ $10^{-10}, U$ test) than responses to the central target. The onset latencies of these responses (mean $1 \lambda, 84 \mathrm{~ms} ; 95 \%$ confidence interval, 75-90 ms; mean $2 \lambda$, 90 ms; confidence interval, $80-100 \mathrm{~ms}$ ) were not significantly different from the latency of the response evoked by the central target (two-sided bootstrap test, $p>0.05$ ). At a distance of $3 \lambda$, flanker responses dropped to a level that was barely different from the baseline ( $p=0.048, U$ test). The onset of responses to the far flankers was significantly later (mean, $109 \mathrm{~ms}$; confidence interval, 90-258 ms) than the onset of the responses to the central target (two-sided bootstrap test, $\left.p<10^{-4}\right)$. These results are consistent with the suggestion that the farthest flankers $(3 \lambda)$ were generally located outside the CRF of the neurons as they elicited very weak responses that occurred later in time than responses to the central target (Bringuier et al., 1999; Li et al., 2000). We note, however, that the extent of the CRF depends on the method used for mapping (Angelucci and Bullier, 2003) and that we cannot exclude that the far flankers fell into the CRF of some of the cells had we measured them with other methods. Figure $2 B$ summarizes the average activity evoked by the central targets and flankers at different separations from the CRF.

Figure $2 C$ shows the population responses to the central Gabor stimuli of tance as well as the tuning of the neuron to spatial frequency (see Materials and Methods). This is attributable to the fact that flanker distance was measured in wavelength $\lambda$, the inverse of spatial frequency. For example, one wavelength corresponds to different contrasts presented either alone or together with the collinear flankers. As expected, the neuronal responses to the central target increased with stimulus contrast. We illustrate the target-evoked responses, Resp $\mathrm{p}_{\text {target }}$, for one of the intermedi- 
A

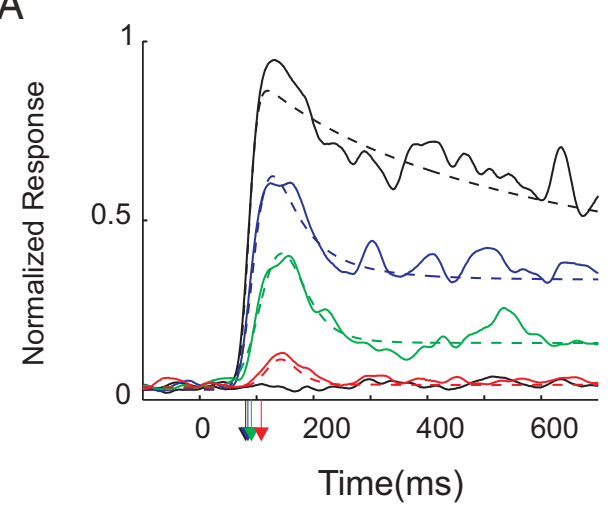

C

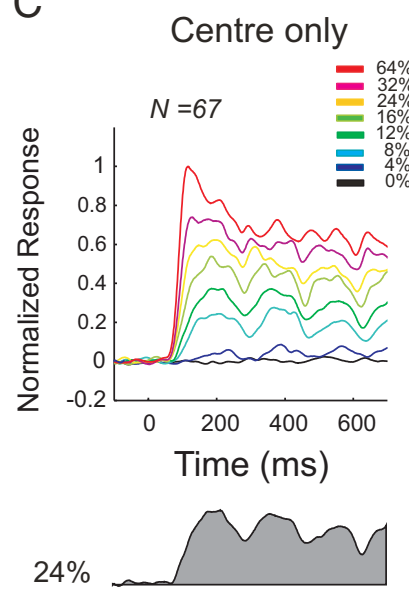

B

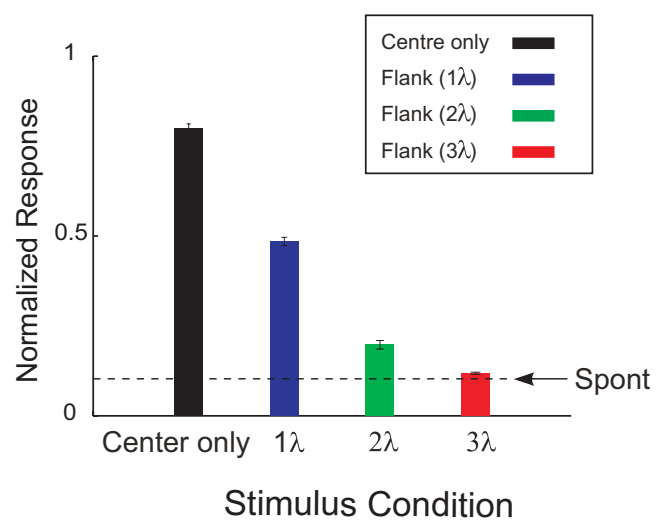

+ Flankers $(2 \lambda)$

+ Flankers $(3 \lambda)$
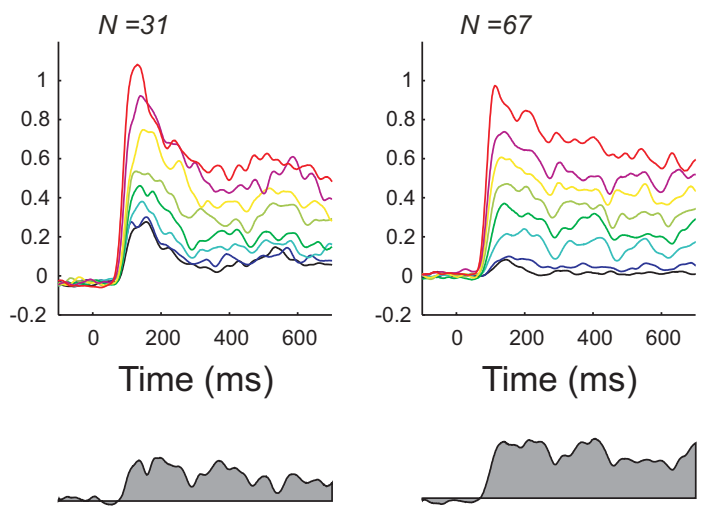

Figure 2. Population analysis of the effects of collinear flankers in area V1. $A$, Comparison of the V1 population responses elicited by the central target to the response evoked by the flanker-only conditions. The dashed lines show the fit to the data used to compute the response latency that was defined as the time that the fitted function reached $33 \%$ of its maximum. The colored arrows on the $x$-axis denote onset latencies. $\boldsymbol{B}$, Average responses in a window from 50 to 350 ms after the stimulus onset. Arrow, 0 ngoing activity in the same time window in the absence of Gabor stimuli. Error bars indicate SEM.C, Population responses evoked by the central stimulus alone or in the presence of the collinear flankers located at three distances (1, 2 , and $3 \lambda$ ). The shaded gray area denotes the responses (Resp target $_{\text {) }}$ ) evoked by a target contrast of $24 \%$, after subtraction of the response evoked by the flankers.

ate stimulus contrast levels $(24 \%)$ in Figure $2 C$ (gray area below $x$-axis). The flankers reduced the target response, and this reduction was most profound at the smaller flanker distances. We measured the Resp $\mathrm{parget}_{\text {as }}$ as the difference between the response to the target plus flankers and to the flankers-only conditions (averaged in a time window from 200 to $700 \mathrm{~ms}$ after the stimulus onset). From these, we constructed the population contrast response functions (Fig. $3 A$ ) and fitted them with a hyperbolic ratio function. The main effect of the collinear flankers was a suppression of the response induced by the center target, which was observed at all contrast levels and at every target-flanker separation. The suppressive effect of the flankers was strongest at the smaller flanker distances. Figure $3 B$ shows the effect of the flankers on the $R_{\text {target_max }}$ and $c_{50}$ across all the recorded neurons, by comparing the responses in the absence (abscissa) or presence (ordinate) of collinear flankers. At distances of 1 and $2 \lambda$, flankers induced a strong suppression indicated by a significant decrease in $R_{\text {target_max }}$ and a significant increase in $c_{50}$ compared with the no-flanker condition (paired $t$ test, all values of $p<0.05$ ). Far flankers $(3 \lambda)$ did not have a significant effect on $R_{\text {target_max }}(p>$ $0.05)$ but caused a highly significant increase in $c_{50}\left(p<5.10^{-6}\right)$. We also investigated whether the amount of suppression depended on the size of the receptive fields of the neurons and the distance of the flankers. Supplemental Figure 1 (available at www. jneurosci.org as supplemental material) shows the receptive fields of all the recorded neurons and the relative distance of the flankers. We did not observe a significant correlation between the amount of suppression and the size of the receptive fields relative to the distance of the flankers. We therefore conclude that variability in the amount of activation of the receptive fields of the neurons by the flankers does not account for the suppressive effects reported here.

Our results so far demonstrate that flankers reduce the neuronal responses evoked by a central target. However, since neuronal responses vary across trials, we also quantified the effects of the flankers with a measure that takes this variability into account. To this end, we measured the detection sensitivity (neuronal $d^{\prime}$ ) of every neuron, which is computed as the difference between the responses to the central stimulus and ongoing activ-

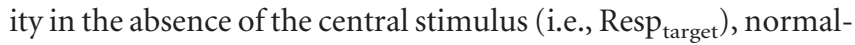
ized to the SD of the response across individual trials (see Materials and Methods). Figure 4 compares the $d^{\prime}$ values of single neurons in the flanker condition with those in the no-flanker condition. At all the target-flanker distances, the majority of data points lie below the line of unity, in accordance with the predominantly suppressive effect of the flankers. We computed the bestfitting regression line to the distribution of $d^{\prime}$ values (Fig. 4, dashed lines) and used the slope to measure the flanker effect on $d^{\prime}$. At all target-flanker distances, the slope of the best-fitting line was $<1$. At a distance of $1 \lambda$, the slope was 0.29 and the $d^{\prime}$ distributions for the center only versus center plus flanker were signif- 
A

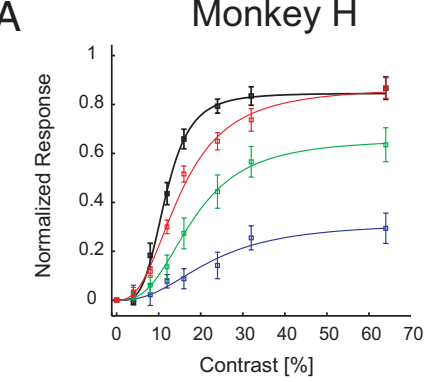

B
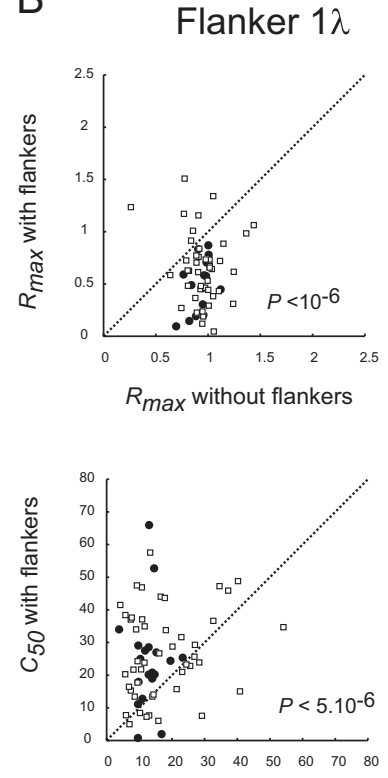

$C_{50}$ without flankers
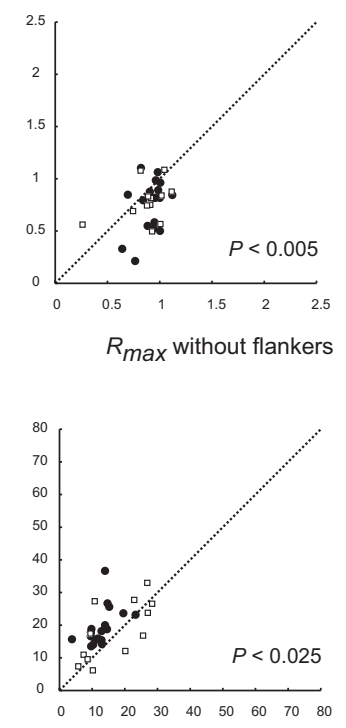

$C_{50}$ without flankers
Monkey D

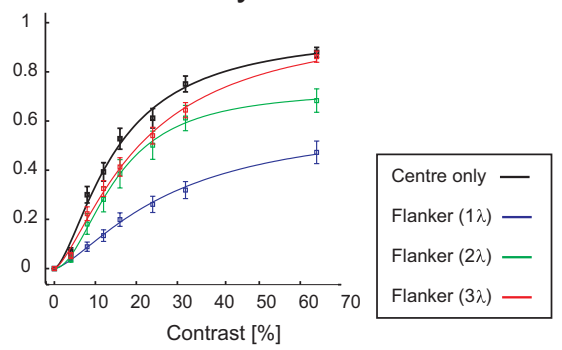

Flanker $2 \lambda$
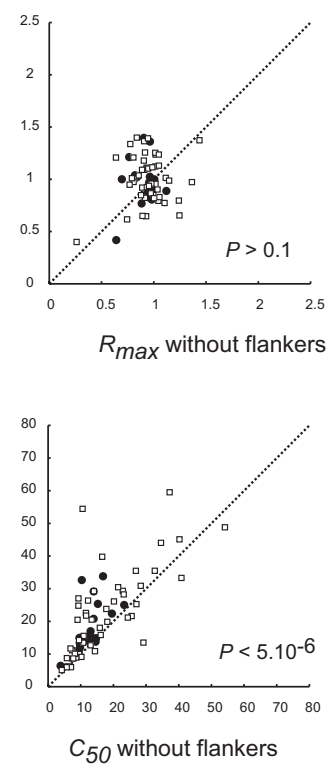

$\square$ Monkey $\mathrm{H}$

- Monkey D

Figure 3. Quantification of flanker modulation at the population level. $A$, Population contrast response functions in area V1. The error bars denote the SEM across the neurons. The curves are hyperbolic ratio functions. $\boldsymbol{B}$, Distribution of $R_{\text {target } \_ \text {max }}$ and $c_{50}$ values derived from the fits to the responses of all V1 single cells. Each point represents $R_{\text {target max }}$ (top panels) or $C_{50}$ (bottom panels) of a single cell in the presence (ordinate) or absence (abscissa) of flankers. The $p$ values are derived from a paired $t$ test.

$\lambda=1$

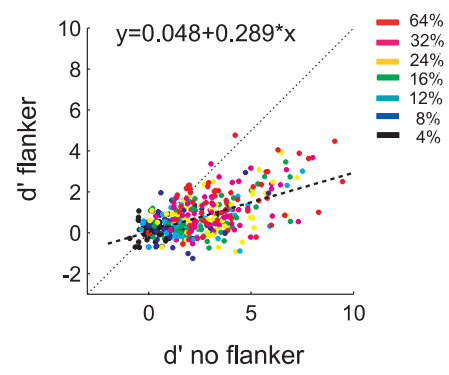

$\lambda=2$

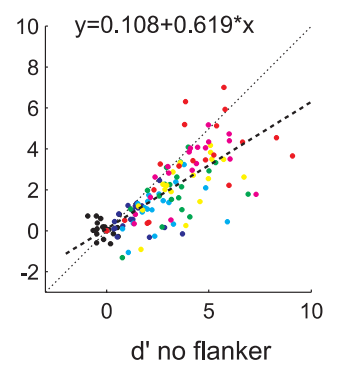

Figure 4. The effects of collinear flankers on neuronal sensitivity. Comparison of the neuronal $d^{\prime}$ values in the presence and in the absence of the collinear flankers. Note that most points lie below the line of unity at all flanker distances and that the slope of the best-fitting regression line is $<1$, indicating that the flankers reduce the neuronal $d^{\prime}$ values.

icantly different ( $p<0.001$, paired $t$ test), the slope increased to 0.62 at $2 \lambda(p<0.001)$, and it increased to 0.82 at $3 \lambda(p>0.5)$.

\section{The effect of collinear flankers on contrast detection}

We were surprised by the consistency of the suppressive effects of collinear flankers across our population of V1 neurons, as this $\lambda=3$

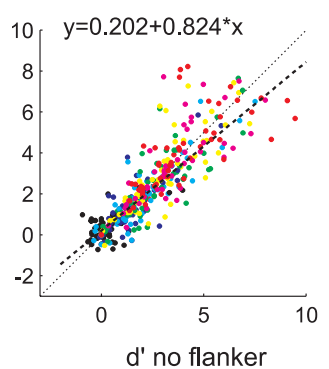

result appears to be in conflict with previous studies that reported predominantly facilitatory effects (Kapadia et al., 1995) or a mixture of facilitatory and suppressive effects (Chen et al., 2001). We considered a number of possibilities for the discrepancy. First, it is possible that we did not observe facilitatory effects of flankers since monkeys were not actively performing a detection task. In the second experiment, we therefore examined the effects of flankers on the performance of monkeys in a contrast detection task, while we simultaneously monitored the activity of $\mathrm{V} 1$ neurons using a multielectrode recording technique. This approach allowed us to directly compare the effects of the flankers on behavior and on neuronal activity, and to sample a larger number of neurons. Second, it is possible that we positioned the flankers too close to the receptive fields of the neurons to observe facilitatory effects. Although the flankers at $3 \lambda$ did not evoke a response in most cells of the first experiment, and $3 \lambda$ was previously shown to be the flanker distance with strongest facilitatory effects (Polat and Sagi, 1993; Williams and Hess, 1998), we tested larger distances between the target and the flankers in the second experiment. Third, we focused on stimuli with lower contrasts and presented the central target later than the flankers to optimize the conditions for facilitation (Polat and Sagi, 2006).

We used a contrast detection task in which the monkeys had to report the presence of a small Gabor target (Fig. 5) (see Materials and Methods). We presented trials with and without flankers in alternating blocks. In trials with flankers, the monkeys first saw a fixation point together with two flanking Gabor elements with a contrast of $13 \%$, whereas they only saw a fixation point in trials without flankers. After a fixation epoch of $500 \mathrm{~ms}$, we presented a Gabor target for $200 \mathrm{~ms}$ on one-half of the trials and no stimulus on the other one-half of trials. After an additional fixation interval of $400 \mathrm{~ms}$ without Gabor elements, the monkeys reported that they saw a target element by making an eye movement to a black circle that was presented at the location of the target or that they did not perceive a target by maintaining fixation.

We will first describe the effect of the flankers on the behavioral performance of the animals (i.e., their effect on contrast sensitivity and the falsealarm rate). Figure $6 \mathrm{~A}$ shows the probability of false alarms, which are reports of seeing the target although it was not there. Flankers increased the probability of false alarms for every targetflanker separation. At a flanker distance of $2.8 \lambda$, monkey A had a false-alarm rate of $14 \%$ without flankers that increased to $18 \%$ in 


\section{Without Flankers}

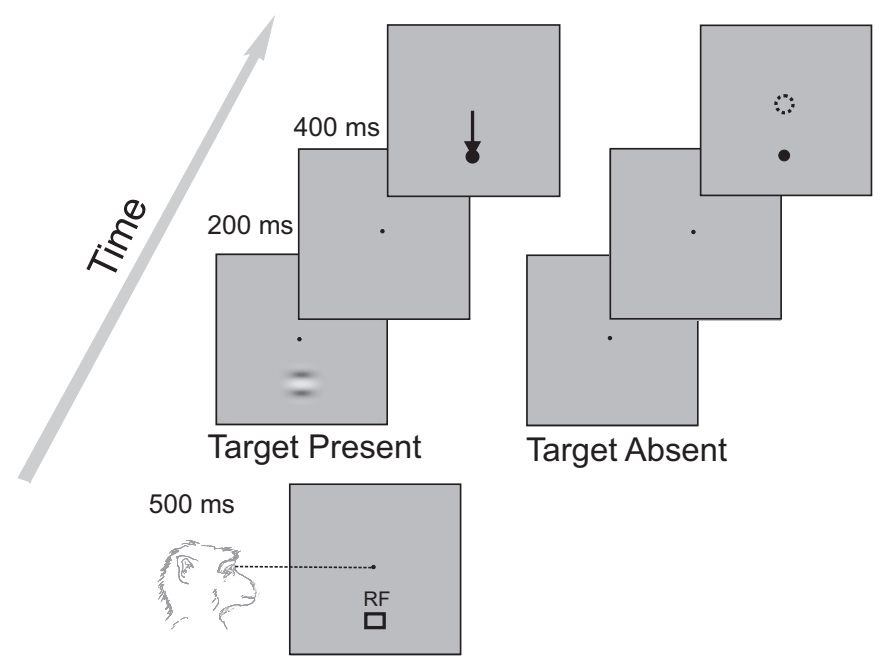

With Flankers
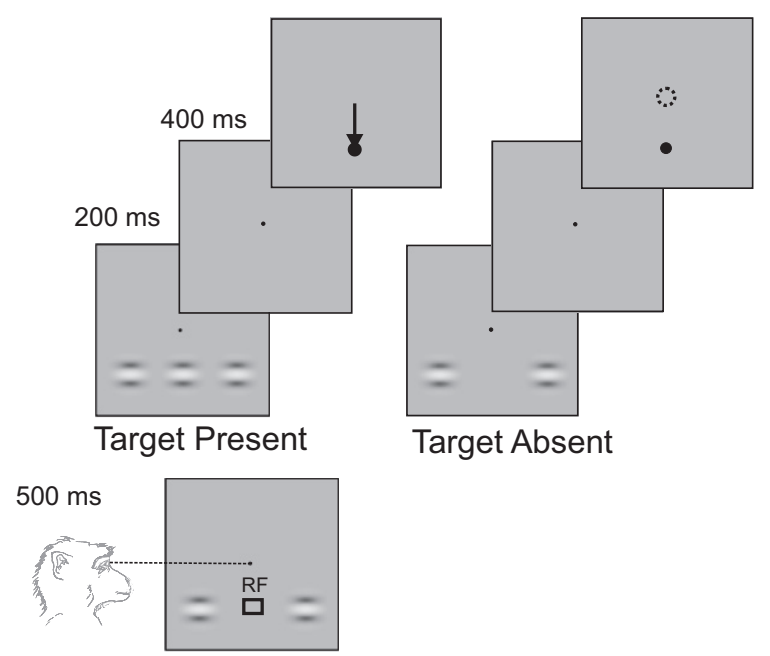

Figure 5. The contrast detection task. The monkeys performed a contrast detection task in the presence or absence of two collinear flankers. We presented collinear flankers or no flankers during the initial fixation interval. After 500 ms of fixation, a Gabor target appeared at the receptive location (RF) for 200 ms or no Gabor stimulus was presented. After an additional 400 ms, the monkey reported his choice by either making an eye movement toward a marker (black circle) at the RF if he had seen the target or by maintaining fixation if not. Six (or seven) different contrast levels (3-20\%) of the central stimulus were tested. Trials with and without the flankers were presented in separate blocks.

the presence of flankers; the false-alarm rate increased from 19 to $22 \%$ at a flanker distance of $3.5 \lambda$ and from 18 to $21 \%$ at a distance of $4.2 \lambda\left(p<10^{-4}\right.$ at every target-flanker distance, two-sided bootstrap test). A similar result was obtained in monkey $\mathrm{G}$ in which the flankers increased the false-alarm rate from 16 to $26 \%$ at a flanker distance of $2.8 \lambda$, from 9 to $16 \%$ at $3.5 \lambda$, and from 18 to $23 \%$ at a distance of $4.2 \lambda$ ( $p<0.01$ at all target-flanker distances, two-sided bootstrap test). We suspect that the relatively high rate of false alarms was related to the inclusion of trials with low-contrast targets $(<10 \%)$ that were difficult to perceive given the short presentation times. These target-present trials presumably induced a low response criterion at the cost of an increased false-alarm rate. We also investigated how the flankers influenced the proportion of hits, trials in which the central target was correctly detected and did not observe consistent effects of the flankers on the hit rate (supplemental Fig. 2, available at www. jneurosci.org as supplemental material).

An increase in the false-alarm rate that is not accompanied by an increase in the hit rate implies that flankers decrease contrast sensitivity. To measure contrast sensitivity while accounting for variations in response criterion, we computed $d^{\prime}$ values (see Materials and Methods). Figure $6 B$ illustrates the $d^{\prime}$ of the monkeys for different contrast levels with and without the collinear flankers. As expected, $d^{\prime}$ generally increased when the stimulus contrast was higher. Monkey A had a lower performance than monkey $\mathrm{G}$ and even missed some of the high-contrast stimuli, which is why we added one extra high-contrast level (60\%) to the stimulus set of this animal, although we limited our analysis to the contrast levels used in both monkeys.

The main effect of collinear flankers was a reduction in $d^{\prime}$, especially at higher stimulus contrasts and at the smaller flanker distances (the significance of difference between $d^{\prime}$ values with and without flankers was tested by a two-sided bootstrap test; the asterisks in Figure $6 B$ indicate a significant difference; $p<0.05$, where $p$ is adjusted for multiple comparisons across six contrast levels). The only exception was for the far flankers in monkey $\mathrm{G}$

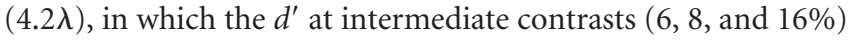
was higher in the presence of flankers, although this increase in performance was not significant. Contrast thresholds (Fig. $6 \mathrm{~B}$, dashed vertical lines) were computed by fitting a Weibull function to the performance data and by measuring the contrast value for which the function reached a $d^{\prime}$ value of 1.5 (i.e., a correct rate of $\sim 70 \%$ if the false alarm is $\sim 15 \%$ ). Collinear flankers significantly increased contrast thresholds at a flanker distance of $2.8 \lambda$ in both monkeys and at 3.5 and $4.2 \lambda$ in monkey A (two-sided bootstrap test, all values of $p<0.05$ ). These results, together, indicate that collinear flankers increased the false-alarm rate of the monkeys and decreased their contrast sensitivity.

\section{The effects of collinear flankers on activity of V1 neurons during behavior}

We will first describe the effects of colinear and orthogonal flankers on neuronal activity measured in a passive fixation task (for details, see Materials and Methods). We compared the magnitude and the latency of the responses evoked by a central Gabor ( $13 \%$ contrast) to the responses evoked by collinear or orthogonal flankers presented alone ( $13 \%$ contrast) and placed at a distance of $2.8,3.2$, and $4.2 \lambda$ from the center of the receptive field (Fig. $7 A, B$ ). Note that, in this passive fixation task, the stimulus (either the central target or the flankers) appeared at time 0 and disappeared after $200 \mathrm{~ms}$. We normalized the responses to the maximum activity evoked by the central Gabor ( $13 \%$ contrast) in a time window from 50 to $150 \mathrm{~ms}$ after the stimulus onset (Fig. $7 A$ ). We averaged responses in a time window between 50 and $350 \mathrm{~ms}$, while normalizing activity to the peak response (Fig. 7A), which is why the average response evoked by the central target was $<1$ (Fig. $7 B$ ). Collinear flankers placed at 2.8 and $3.5 \lambda$ from the CRF center elicited neuronal responses that were significantly higher ( $p<0.01$, Mann-Whitney $U$ test) than the baseline, but weaker ( $p<0.01$, Mann-Whitney $U$ test) and delayed ( $p<0.05$, two-sided bootstrap test) compared with the responses to the central Gabor (onset latencies: central target, $58 \mathrm{~ms}$; flankers at $2.8 \lambda, 70 \mathrm{~ms}$; flankers at $3.5 \lambda, 71 \mathrm{~ms}$ ). Responses evoked by the collinear flankers decreased at larger distances from the CRF, and the flankers did not evoke a significant response if they were at a distance of $4.2 \lambda$ ( $p>0.05$, Mann-Whitney $U$ test). Neuronal 
A

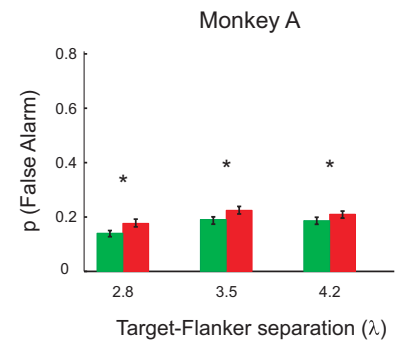

$2.8 \lambda$
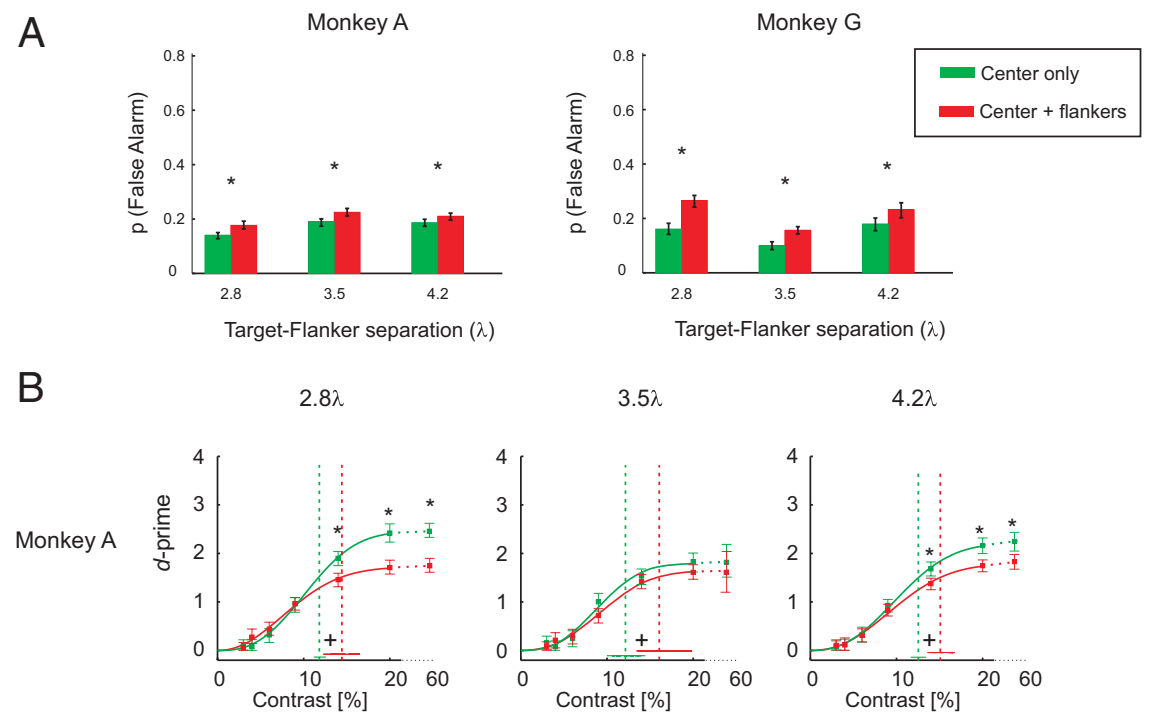

B

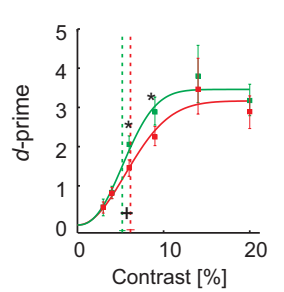

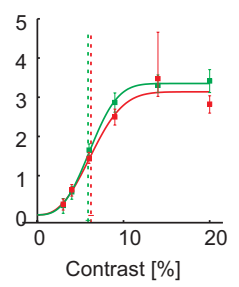

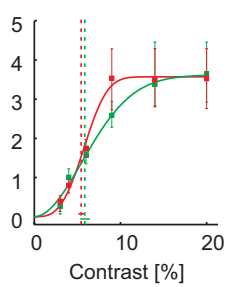

Figure 6. Behavioral performance. $\boldsymbol{A}$, Probability of false alarms with (red bars) and without flankers (green bars) in the two monkeys. Note that the various flanker distances were tested on different days and that the green bars denote false-alarm rates in the corresponding sessions for the no-flanker trials. The error bars indicate the $95 \%$ confidence intervals determined with a bootstrapping test. The asterisks denote significant differences $(p<0.05)$ in FA rate between trials with and without flankers measured by a two-sided bootstrap test. $\boldsymbol{B}$, Detection sensitivity $\left(d^{\prime}\right)$ at different contrast levels with and without the flankers. The vertical dashed lines represent the behavioral contrast thresholds. Error bars represent the $95 \%$ confidence intervals. A plus $(+)$ sign indicates that the contrast thresholds with and without the flankers were significantly different. The asterisks $\left({ }^{*}\right)$ denote that the $d^{\prime}$ values with and without the flankers were significantly different (two-sided bootstrap test, $p<0.05$ with Bonferroni's correction for multiple comparisons).

responses to the orthogonal flankers at 2.8 and $3.5 \lambda$ were significantly weaker ( $p<0.01, U$ test) than responses to the collinear flankers at the same location but had a similar latency (flankers at $2.8 \lambda, 66 \mathrm{~ms}$; flankers at $3.5 \lambda, 78 \mathrm{~ms} ; p>0.05$, two-sided bootstrap test). At a distance of $4.2 \lambda$, responses to the orthogonal flankers became indistinguishable from the baseline $(p>0.05)$.

The activity induced by the collinear flankers depended on the preferred orientation of the recording sites. The orientation tuning of the recording sites was determined by measuring the responses to a moving bar stimulus (see Materials and Methods). We measured the orientation selectivity of the recording sites by computing an orientation selectivity ratio (OSR), defined as the ratio between the response to the best and the worst orientations, after subtraction of the baseline activity. OSRs ranged from 1.2 to 4.3 with a median of $1.7(N=38)$. For every recording site, we determined how well the horizontal orientation used in our experiments matched the preferred orientation of that recording site, defining the following three categories: $0-30,30-60$, and $60-90^{\circ}$ from the horizontal orientation. Figure $7 C$ shows the responses evoked by the collinear flankers at these three groups of recording sites in the absence of a target stimulus, normalized to the responses evoked by the horizontal stimulus centered on the RF. At all target-flanker distances, the responses to the collinear flankers were highest when they matched the preferred orienta- tion of the recording site, and they were at an intermediate level when the orientation of the flankers differed by $30-60^{\circ}$ from the preferred orientation and weakest when the flankers were approximately orthogonal to the preferred orientation. The significance of this effect was confirmed with an $\operatorname{ANOVA}\left(F_{(2,87)}=6.2 ; p=\right.$ 0.003 ) with factors flanker distance (three distances) and the orientation of the flankers relative to the preferred orientation of the recordings sites.

We next examined the neuronal responses while monkeys performed the detection task (Fig. 5). Figure 7D illustrates the population responses (average across 38 recording sites) evoked by the central target alone as well as by the target plus collinear flankers at the three flanker separations. These data were normalized to the $20 \%$ contrast stimulus in the noflanker condition that was repeated on every recording day, but as different target-flanker distances were tested in different recording sessions the data of the various flanker distances are shown in separate panels. The flankers that were presented $500 \mathrm{~ms}$ before the target (i.e., at $t=-500 \mathrm{~ms}$ in Fig. 7D) caused an increase in activity even in the absence of the target (note the gray areas in Fig. 7D) that was strongest if they were close to the CRF. As expected, the strength of the response evoked by the central Gabor target increased with stimulus contrast. To compute the responses that were evoked by the central target $\left(\right.$ Resp $\left.\mathrm{p}_{\text {target }}\right)$, we subtracted the responses in target-absent trials from the responses in target-present trials. Figure $7 D$ shows an example of the responses evoked by a $9 \%$ contrast target (yellow area depicts Resp target ). It can be seen that the flankers decreased the responses to the central target, because these responses had to be evoked on top of Resp flankers (Fig. 7D, gray area).

Figure $8 \mathrm{~A}$ illustrates the effect of the flankers on responses evoked by the target in the time window from 50 to $350 \mathrm{~ms}$ after target appearance for the various contrast levels. In both monkeys, the flankers at every distance decreased the magnitude of the response evoked by the target. The suppressive effect of flankers was largest at small distances and for targets with higher contrasts. Similar to our single-cell experiments, we quantified the flanker effect by fitting a hyperbolic ratio function to the contrast response curve of every recording site (Fig. $8 \mathrm{~B}$ ). The flankers significantly decreased the $R_{\text {target_max }}$ values at all distances (paired $t$ test, all values of $p<0.001$ ) compared with the noflanker condition. Closer flankers $(2.8 \lambda)$ also significantly increased the $c_{50}$ values (paired $t$ test, $p<0.01$ ), but at larger flanker distances the increase in $c_{50}$ was not statistically significant. Therefore, the major effect of flankers in the contrast detection task was a decrease in the $R_{\text {target_max }}$.

To examine the distribution of the modulatory effect of flankers across the population of V1 neurons, we computed the neuronal $d^{\prime}$ for every individual recording site. In Figure $9 A$, 

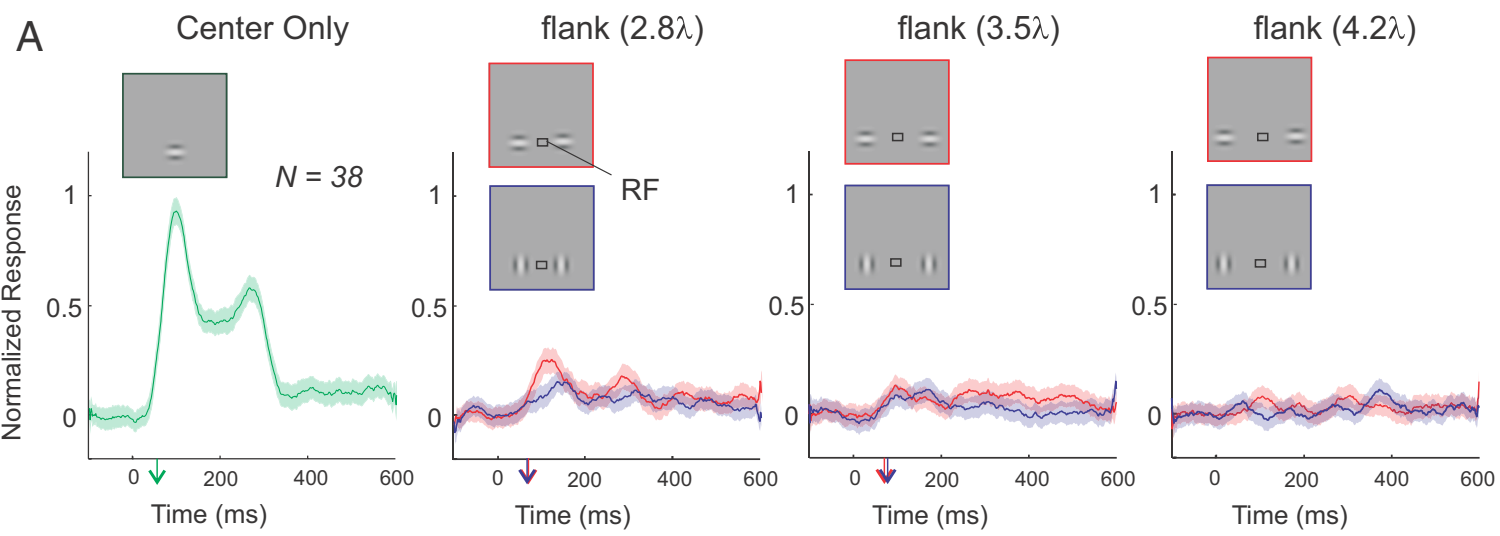

B
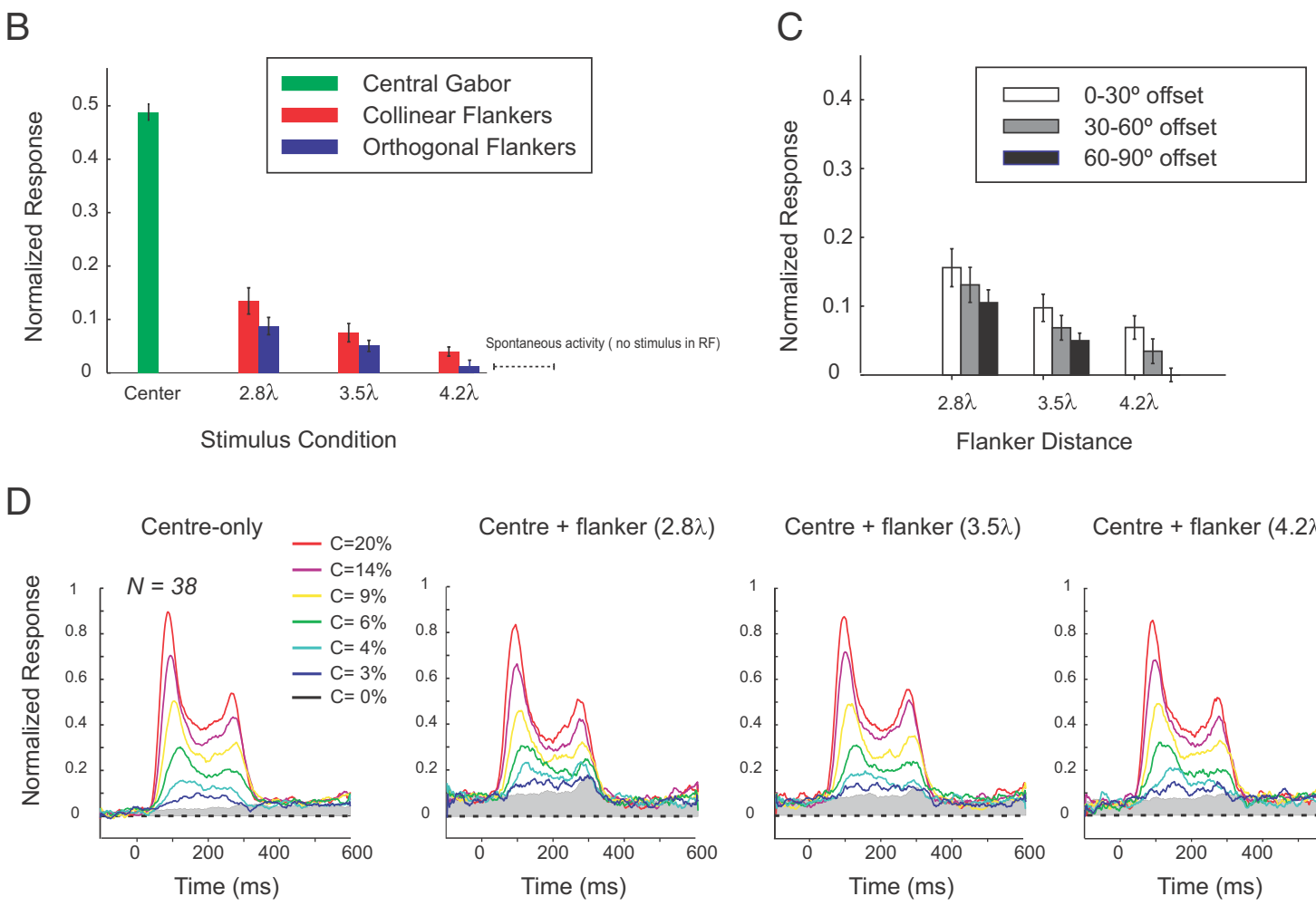

Centre + flanker $(3.5 \lambda)$

Centre + flanker $(4.2 \lambda)$
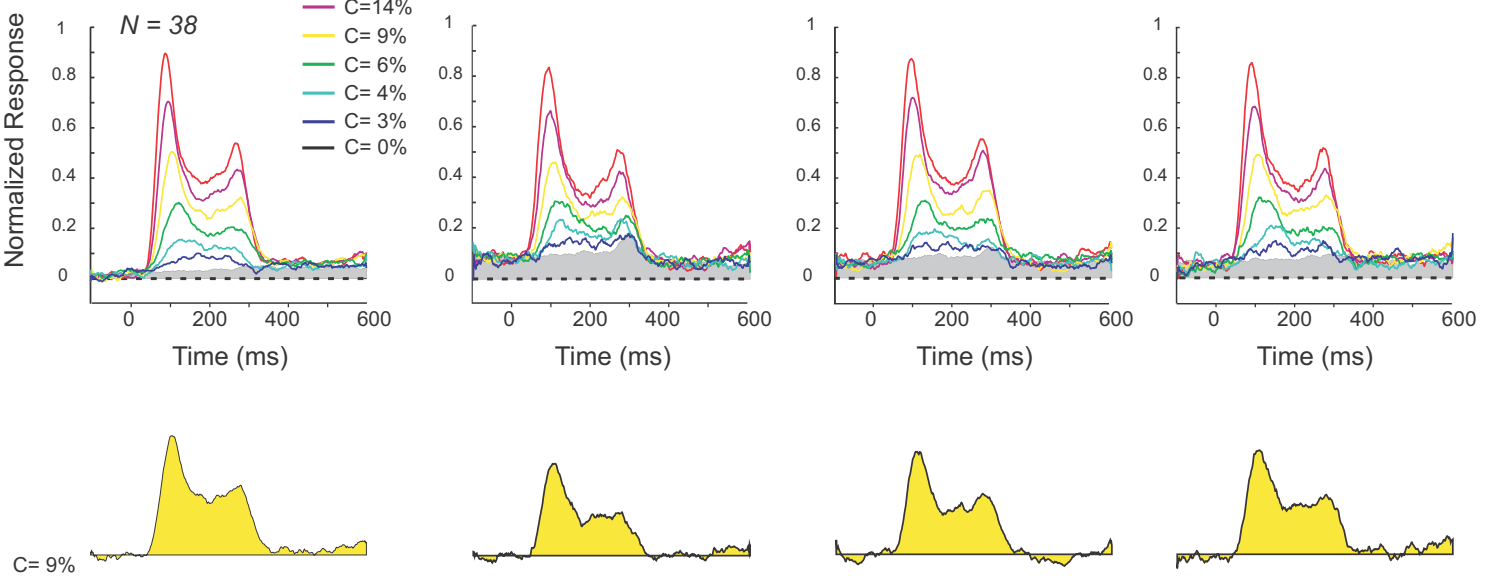

Figure 7. Effects of flankers on the population responses in area V1. $A$, The neuronal responses evoked by a central target (green) are compared with the responses elicited by the collinear (red) and orthogonal flankers (blue) placed at three distances $(2.8,3.5$, and $4.2 \lambda$ ). The arrows on the $x$-axis denote the response latencies. $\boldsymbol{B}$, Average neuronal activity in a time window from 50 to 350 ms after stimulus onset (same data as in $A$ ). C, Activity induced by collinear flankers as a function of the difference between the presented and preferred orientation $\left(0-30,30-60\right.$, and $60-90^{\circ}$ ). Error bars indicate SEM. $\boldsymbol{D}$, Responses evoked by the central target at different contrast levels. The yellow response shown below represents the difference between the activity evoked by a central Gabor with $9 \%$ contrast in the presence of flankers and the activity evoked by the flankers on target-absent trials. Note that the flankers reduce the response. Gray area in the upper panels shows neuronal activity in the absence of the central target stimulus.

neuronal $d^{\prime}$ values in the presence of the flankers (ordinate) are plotted against neuronal $d^{\prime}$ values in target-only condition (abscissa). Each point represents the neuronal $d^{\prime}$ of an individual recording site at a certain contrast level. For all target-flanker distances, the data points lie below the line of unity indicating that collinear flankers decreased the neuronal sensitivity. The slope of the best-fitting regression line is an indicator of the direction and magnitude of the flanker effect and it was invariably $<1$ (neuronal $d^{\prime}$ values without flankers $>$ neuronal $d^{\prime}$ values with flankers; all values of $p<0.01$, paired $t$ test). The data were well fitted by a line, which suggests that the flankers decreased neuronal sensitivity by a constant fraction. Moreover, the decrease in $d^{\prime}$ was most pronounced at smaller target-flanker separations.

We used a single stimulus for multiple recording sites and we therefore performed a control analysis to investigate whether the suppressive effects were driven by those recording sites where the flankers were relatively close to the RF. However, we found that 
A $2.8 \lambda$
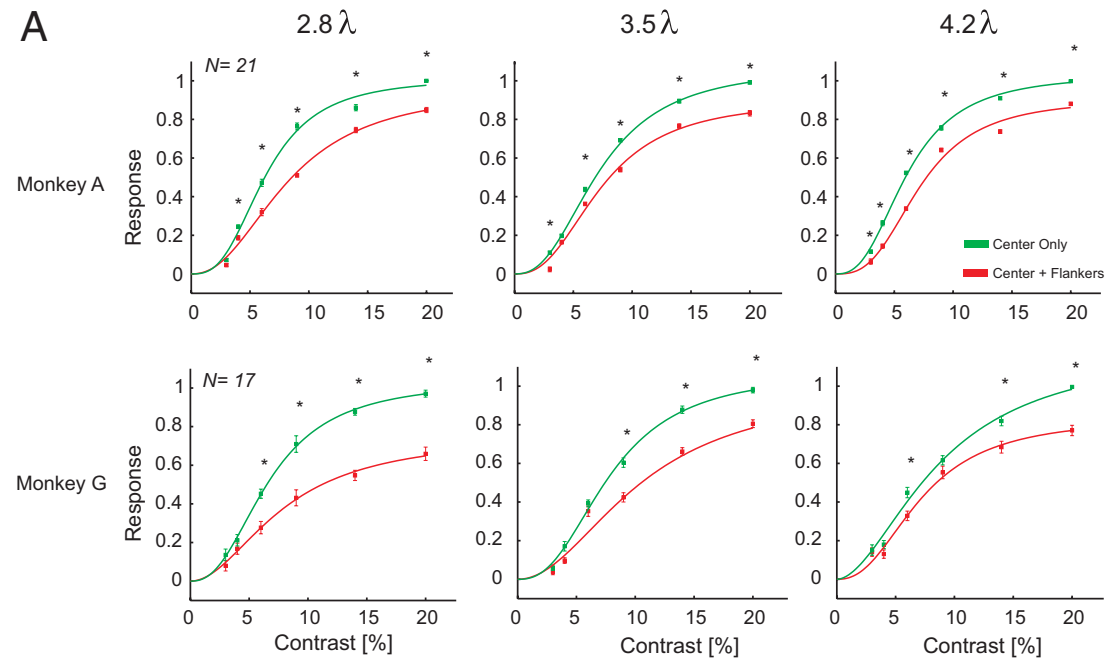

B
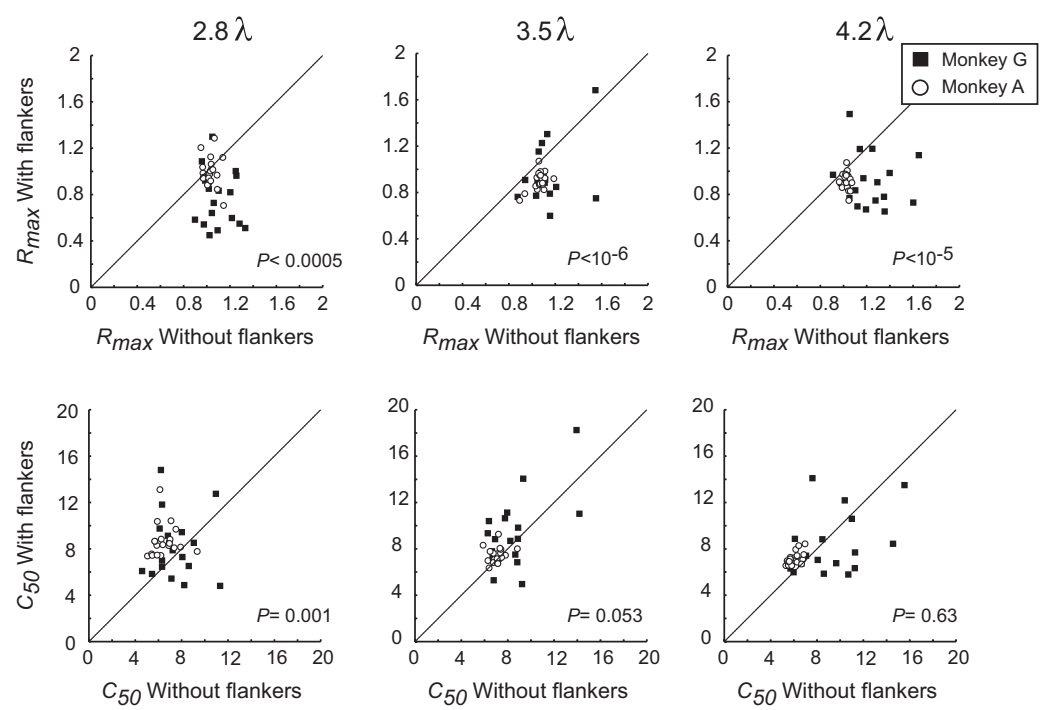
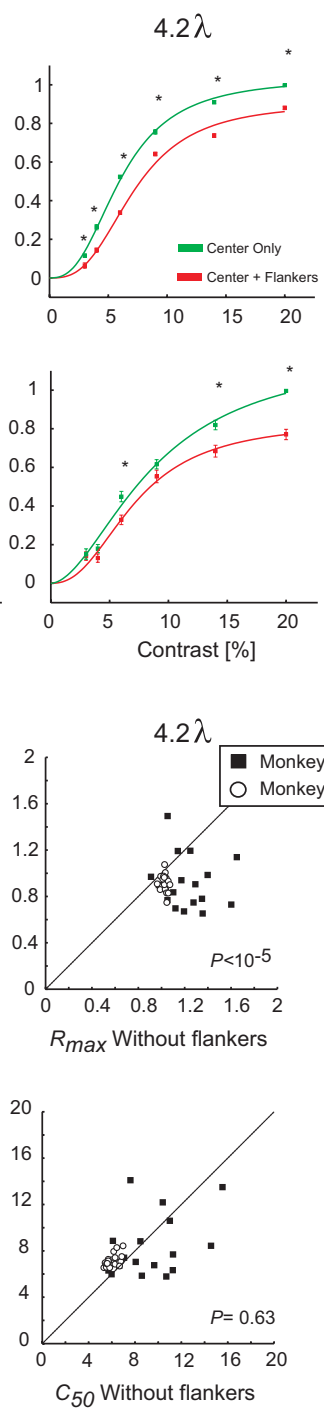

Figure 8. Quantification of flanker modulation in a population of V1 recording sites. $A$, Contrast response functions averaged across all recording sites in the presence and absence of the flankers. The error bars represent the SEM. The asterisks $\left({ }^{*}\right)$ denote a significant difference in the response evoked by the target in the presence and absence of flankers ( $p<0.05$, Mann-Whitney $U$ test with Bonferroni's correction for multiple comparisons). $\boldsymbol{B}$, Distribution of $R_{\text {target max }}$ and $c_{50}$ values derived from a hyperbolic fit to the responses of all $\mathrm{V} 1$ recording sites in the presence (ordinate) or absence (abscissa) of flankers. The values of $p$ are computed with a paired $t$ test.

the RFs were generally well centered on the central Gabor (in accordance with the responses shown in Fig. 7A) and we did not observe a significant correlation between the distance between the flankers and the RF and the decrease in contrast sensitivity of the neurons (supplemental Fig. 3, available at www.jneurosci.org as supplemental material). An additional control analysis showed that the distributions of $X$ and $Y$ eye positions were not significantly influenced by the presence of flankers ( $t$ test, both values of $p>0.4)$ and that difference between the average eye position for stimuli with and without flankers was $<0.01^{\circ}$. We also investigated the possibility that inaccurate fixation caused the flankers to enter into the RF and that this contributed to the suppression. We compared the suppression on trials with accurate fixation to trials with less accurate fixation and found that suppression was strong in both types of trials (supplemental Fig. 4, available at www.jneurosci.org as supplemental material). Thus, the suppression was not caused by trials with less accurate fixation.

\section{Comparison of flanker effects on neuronal $d^{\prime}$ between experiments}

We used larger flanker separations in the contrast detection task than in the first experiment and recorded multiunit activity rather than single units. Are the effects of flankers comparable across the two experiments? To address this question, we computed the slope of the regression lines for the effect of the flankers on neuronal $d^{\prime}$ (Figs. 4, 9A). In all instances, collinear flankers resulted in a suppression of the neuronal $d^{\prime}$ values (Fig. 9B). In the first experiment, the $d^{\prime}$ was reduced to 29,62 , and $82 \%$ of the value in the no-flanker condition for flankers at a distance of 1,2 , and $3 \lambda$, respectively, whereas the $d^{\prime}$ of the second experiment was reduced to 81,90 , and $91 \%$ for distances of $2.8,3.5$, and $4.2 \lambda$, respectively. It can be seen that the flankers at similar distances evoked a comparable degree of suppression of neuronal sensitivity in the two experiments. Furthermore, we investigated the effect of using different time windows in the two experiments: $200-700 \mathrm{~ms}$ in the singleunit experiments and 50-350 $\mathrm{ms}$ in the multiunit experiments. To increase comparability between data sets further, we reanalyzed the single-unit data in a 50-350 ms window and obtained virtually identical results with those reported for the 200-700 ms analysis (supplemental Fig. 4, available at www.jneurosci.org as supplemental material).

\section{Discussion}

We have found that collinear flankers at distances that have commonly been associated with an increase in contrast sensitivity induced a decrease in the contrast sensitivity of neurons in area V1. When a central target was presented together with collinear flankers, the response to the target plus flankers was equal to or less than the response evoked by the target alone. Flankers at distances up to $3.5 \lambda$ increased the ongoing activity of V1 neurons and the response evoked from the enhanced baseline activity was smaller than the response evoked by the same target in the absence of flankers. This decreased responsiveness caused a decrease in the neuronal $d^{\prime}$ and was also accompanied by a decrease in contrast sensitivity at the behavioral level.

At first sight, these results are at odds with psychophysical experiments showing that flankers improve contrast detection and also with previous neurophysiological studies reporting mainly facilitatory flanker effects (Kapadia et al., 1995; Polat et al., 1998; Chen et al., 2001; Kasamatsu et al., 2001; Mizobe et al., 2001; Crook et al., 2002). Why did we find suppression where previous studies tended to observe facilitation? We will we first address the effects of the flankers on the ongoing activity and then discuss the possible reasons for the suppressive effects of the flankers. 


\section{Effects of flankers on the false-alarm rate and ongoing neuronal activity} Collinear flankers increased the probability of false alarms (Fig. 6A). This result is reminiscent of a recent study in human observers showing that flankers increase the false-alarm rate, an effect that is particularly strong if trials with and without flankers are interleaved (Polat and Sagi, 2007). Polat and Sagi (2007) speculated that the false alarms are caused by a perceptual process that fills in the space between the flankers. They suggested that this process might induce activity in neurons with receptive fields on the background region between the flankers (Meng et al., 2005). In their study, the increase in the false-alarm rate was smaller if the flanker and no-flanker conditions were presented in different blocks so that subjects could adjust their response criterion for the central target, and could be more conservative in reporting targets in the presence of the flankers. However, even if the flanker and no-flanker trials were presented in different blocks, flankers induced extra false alarms, just as we observed in the present study. In accordance with the suggestion of Polat and Sagi (2007), we found that the flankers presented at distances $<3.5 \lambda$ increased the activity of neurons with a receptive field between the flankers. Collinear Gabor elements induced a stronger increase of the ongoing activity than orthogonal flankers, which is in accordance with the finding that noncollinear flankers also cause a smaller increase in the false-alarm rate in human observers (Polat and Sagi, 2007).

We chose flanker distances that induce facilitation in human foveal vision and we were not surprised to find an effect on "ongoing" activity for the shorter distances ( 1 and $2 \lambda$ ) in which flankers probably encroached on the CRF in many of our experiments. Flankers at $3 \lambda$ and larger distances caused only a weak effect on the ongoing activity, and this effect was strongest for collinear flankers. We do not claim, however, that the flankers at a distance of $3 \lambda$ were always entirely outside the CRF for at least two reasons. First, the boundary of the CRF can be defined in a number of ways (Angelucci and Bullier, 2003) and the receptive field size has been shown to be larger for stimuli with a lower contrast (Kapadia et al., 1999; Sceniak et al., 1999). Second, if the receptive field size is determined with pairs of flanker bars symmetrically arranged across the CRF center, the observed CRF is larger than when it is mapped with more standard techniques (Angelucci and Bullier, 2003). Nevertheless, we found that the activity evoked by flankers farther from the CRF center was weaker and had a later onset compared with the responses elicited by the central target, in line with previous findings (Li et al., 2000). This delay has been proposed to reflect the horizontal propagation of the signals from the adjacent neurons (Bringuier et al., 1999), although some authors have suggested that the dynamics of surround effects better match with the feedback signals from extrastriate cortex (Angelucci and Bullier, 2003). It is also possible that the weaker response and increased delay is caused by less efficient feedforward input when the flankers are placed outside the central CRF causing an extra delay before the neurons reach firing threshold.

\section{Effects of flankers on neuronal contrast sensitivity}

In the presence of the flankers, the center target had to evoke a response on top of the enhanced baseline firing rate. The response to maximal contrast targets, however, remained the same or was even reduced compared with center target presentation only. The flankers therefore decreased the dynamic range $\left(R_{\max }\right)$ of the V1 responses. This decrease was larger for targets with higher contrasts (Figs. 3, $8 \mathrm{~A}$ ). Accordingly, the neuronal $d^{\prime}$ values were reduced by a fraction that was relatively constant for a specific flanker separation (Figs. 4, 9A). The magnitude of this fractional decrease in $d^{\prime}$ was largest for flankers closest to the central Gabor element (Fig. 9B). In addition, the flankers increased $c_{50}$, in particular at the smaller distances $(<3.5 \lambda)$, corresponding to a rightward shift of the contrast response function.

The finding of a decrease in neuronal contrast sensitivity appears to be in conflict with previous studies that uncovered facilitatory flanker effects (Kapadia et al., 1995), especially at the lower contrasts (Polat et al., 1998), or a mixture of facilitatory and inhibitory effects (Kapadia et al., 2000; Kasamatsu et al., 2001; Mizobe et al., 2001), although we also found facilitatory interactions in a small number of cells (Fig. 1D). Why did we find such a predominance of suppressive effects, whereas previous studies found much more evidence for facilitation? One possible explanation is that we fixed the distances between the flanker and central targets at multiples of the Gabor wavelength $(\lambda)$, in accordance with the procedures used in most psychophysical studies, whereas previous neurophysiological studies usually ensured that the flankers did not induce any response above the baseline. We note, however, that we also observed a small but highly consistent suppression at the largest flanker distance of $4.2 \lambda$ that did not induce a significant increase in the baseline firing rate. Another possibility is that we used Gabor stimuli, whereas the studies that 
observed a predominance of facilitatory effects used short light bars (Kapadia et al., 1995, 1999, 2000). However, we do not consider this possibility likely because mixtures of facilitatory and suppressive effects were also observed in studies in anesthetized cats that used Gabor stimuli (Chen et al., 2001; Kasamatsu et al., 2001; Mizobe et al., 2001; Crook et al., 2002). Furthermore, our results fit well with a recent study that used short bars and measured the effects of flankers in awake monkeys with optical imaging of intrinsic signals (Kinoshita et al., 2009). This study demonstrated that flankers caused a robust reduction in the intrinsic signals evoked by targets of all contrasts. Moreover, we performed an additional experiment with short bar stimuli and observed predominantly suppressive effects (supplemental Fig. 5, available at www.jneurosci.org as supplemental material). Another recent study using voltage-sensitive dye imaging found that Gabor flankers reduce the subthreshold neuronal responses evoked by a low-contrast Gabor target (Meirovithz et al., 2010). We therefore feel confident about the predominance of suppressive effects of the flankers on contrast sensitivity of spiking activity in area V1 that was observed by us in two experiments with different recording techniques. The present study is the first to measure the effect of flankers on behavioral contrast sensitivity in monkeys during the recordings. We also observed a suppression of contrast sensitivity at the behavioral level, in accordance with the effects on the neuronal activity in area V1.

Even stronger suppressive effects are obtained with abutting collinear bars that are displaced in the direction of the short axis (i.e., next to the target bar) (Macknik and Livingstone, 1998). These abutting flankers produce suppression when they are presented before (forward masking), after (backward masking), or at the same time (simultaneous masking), and produce masking effects that can render the target bar completely invisible (Martinez-Conde et al., 2002; Macknik and Martinez-Conde, 2004).

\section{Flanker effects on contrast sensitivity in behavior}

In human psychophysics, the predominant effect of collinear flankers is an increase of the sensitivity for low-contrast targets (Polat and Sagi, 1994; Morgan and Dresp, 1995; Wehrhahn and Dresp, 1998; Chen and Tyler, 2002). We observed that the flankers reduced the contrast sensitivity in monkeys, with largest decreases in sensitivity for the smaller flanker distances. Our findings therefore appear in conflict with previous psychophysical findings. However, the reduction in contrast sensitivity observed here is in line with human psychophysical work that compared flanker effects in the fovea with their effects at more peripheral locations in the visual field. Although flankers increase the contrast sensitivity of human observers at the fovea, they have less of an effect or decrease contrast sensitivity at parafoveal and more eccentric locations (Williams and Hess, 1998; Macknik et al., 2000; Zenger-Landolt and Koch, 2001; Shani and Sagi, 2005; Macknik, 2006).

Two hypotheses have been proposed to account for the effect of eccentricity on flanker facilitation. The first holds that subjects allocate less attention to the flankers at eccentric locations than when they are presented close to the fovea. This view received support from findings that the facilitatory effects can be restored by directing attention to the flankers in the periphery (Giorgi et al., 2004; Shani and Sagi, 2005), which is in accordance with a study showing that flanker effects at the fovea also depend on attention (Freeman et al., 2001) and also with the findings of Roberts et al. (2007), who showed that attention increases spatial integration at peripheral locations.
The second hypothesis is that the interactions between adjacent V1 neurons in the fovea and the periphery are fundamentally different, with stronger facilitation at the fovea and stronger inhibitory interactions in the periphery (Xing and Heeger, 2000; Zenger-Landolt and Koch, 2001; Petrov et al., 2005). This view is supported by "pathfinder" studies in which subjects have to detect a string of collinearly aligned Gabor elements in a background of elements with random orientations. The efficiency of this pathfinding process is much lower in the periphery of the visual field than at the fovea (Hess and Dakin, 1997; Hess and Field, 1999), in accordance with the suggestion that lateral interactions in the periphery and at the fovea have different properties.

In conclusion, our results indicate that the predominant effect of collinear flankers at parafoveal locations is suppressive. Flankers cause an increase of the ongoing activity that scales down the activity evoked by a central target, and these suppressive effects are accompanied by a reduction of contrast sensitivity at the behavioral level. Future neurophysiological studies could compare the flanker effects at foveal locations with those in the periphery to further bridge the gap between the neurophysiology and the psychophysics of interactions between the representations of adjacent image elements in early vision.

\section{References}

Adini Y, Sagi D (2001) Recurrent networks in human visual cortex: psychophysical evidence. J Opt Soc Am A Opt Image Sci Vis 18:2228-2236.

Albrecht DG, Hamilton DB (1982) Striate cortex of monkey and cat: contrast response function. J Neurophysiol 48:217-237.

Albright TD, Stoner GR (2002) Contextual influences on visual processing. Annu Rev Neurosci 25:339-379.

Angelucci A, Bullier J (2003) Reaching beyond the classical receptive field of V1 neurons: horizontal or feedback axons? J Physiol Paris 97:141-154.

Bour LJ, van Gisbergen JA, Bruijns J, Ottes FP (1984) The double magnetic induction method for measuring eye movement-results in monkey and man. IEEE Trans Biomed Eng 31:419-427.

Bringuier V, Chavane F, Glaeser L, Frégnac Y (1999) Horizontal propagation of visual activity in the synaptic integration field of area 17 neurons. Science 283:695-699.

Chen CC, Tyler CW (2002) Lateral modulation of contrast discrimination: flanker orientation effects. J Vis 2:520-530.

Chen CC, Kasamatsu T, Polat U, Norcia AM (2001) Contrast response characteristics of long-range lateral interactions in cat striate cortex. Neuroreport 12:655-661.

Crook JM, Engelmann R, Löwel S (2002) GABA-inactivation attenuates colinear facilitation in cat primary visual cortex. Exp Brain Res 143:295-302.

Das A, Gilbert CD (1999) Topography of contextual modulations mediated by short-range interactions in primary visual cortex. Nature 399:655-661.

DeAngelis GC, Freeman RD, Ohzawa I (1994) Length and width tuning of neurons in the cat's primary visual cortex. J Neurophysiol 71:347-374.

Freeman E, Sagi D, Driver J (2001) Lateral interactions between targets and flankers in low-level vision depend on attention to the flankers. Nat Neurosci 4:1032-1036.

Gilbert CD (1998) Adult cortical dynamics. Physiol Rev 78:467-485.

Giorgi RG, Soong GP, Woods RL, Peli E (2004) Facilitation of contrast detection in near-peripheral vision. Vision Res 44:3193-3202.

Gorea A, Caetta F, Sagi D (2005) Criteria interactions across visual attributes. Vision Res 45:2523-2532.

Hess R, Field D (1999) Integration of contours: new insights. Trends Cogn Sci 3:480-486.

Hess RF, Dakin SC (1997) Absence of contour linking in peripheral vision. Nature 390:602-604.

Ito M, Gilbert CD (1999) Attention modulates contextual influences in the primary visual cortex of alert monkeys. Neuron 22:593-604.

Kapadia MK, Ito M, Gilbert CD, Westheimer G (1995) Improvement in visual sensitivity by changes in local context: parallel studies in human observers and in V1 of alert monkeys. Neuron 15:843-856.

Kapadia MK, Westheimer G, Gilbert CD (1999) Dynamics of spatial sum- 
mation in primary visual cortex of alert monkeys. Proc Natl Acad Sci U S A 96:12073-12078.

Kapadia MK, Westheimer G, Gilbert CD (2000) Spatial distribution of contextual interactions in primary visual cortex and in visual perception. J Neurophysiol 84:2048-2062.

Kasamatsu T, Polat U, Pettet MW, Norcia AM (2001) Colinear facilitation promotes reliability of single-cell responses in cat striate cortex. Exp Brain Res 138:163-172.

Kinoshita M, Gilbert CD, Das A (2009) Optical imaging of contextual interactions in V1 of the behaving monkey. J Neurophysiol 102:1930-1944.

Lamme VA, Zipser K, Spekreijse H (1998) Figure-ground activity in primary visual cortex is suppressed by anesthesia. Proc Natl Acad Sci U S A 95:3263-3268.

Li W, Thier P, Wehrhahn C (2000) Contextual influence on orientation discrimination of humans and responses of neurons in V1 of alert monkeys. J Neurophysiol 83:941-954.

Macknik SL (2006) Visual masking approaches to visual awareness. Prog Brain Res 155:177-215.

Macknik SL, Livingstone MS (1998) Neuronal correlates of visibility and invisibility in the primate visual system. Nat Neurosci 1:144-149.

Macknik SL, Martinez-Conde S (2004) Dichoptic visual masking reveals that early binocular neurons exhibit weak interocular suppression: implications for binocular vision and visual awareness. J Cogn Neurosci 16:1049-1059.

Macknik SL, Martinez-Conde S, Haglund MM (2000) The role of spatiotemporal edges in visibility and visual masking. Proc Natl Acad Sci U S A 97:7556-7560.

Martinez-Conde S, Macknik SL, Hubel DH (2002) The function of bursts of spikes during visual fixation in the awake primate lateral geniculate nucleus and primary visual cortex. Proc Natl Acad Sci U S A 99:13920-13925.

Meirovithz E, Ayzenshtat I, Bonneh YS, Itzhack R, Werner-Reiss U, Slovin H (2010) Population response to contextual influences in the primary visual cortex. Cereb Cortex 20:1293-1304.

Meng M, Remus DA, Tong F (2005) Filling-in of visual phantoms in the human brian. Nat Neurosci 8:1248-1254.

Mizobe K, Polat U, Pettet MW, Kasamatsu T (2001) Facilitation and suppression of single striate-cell activity by spatially discrete pattern stimuli presented beyond the receptive field. Vis Neurosci 18:377-391.

Morgan MJ, Dresp B (1995) Contrast detection facilitation by spatially separated targets and inducers. Vision Res 35:1019-1024.

Petrov Y, Carandini M, McKee S (2005) Two distinct mechanisms of suppression in human vision. J Neurosci 25:8704-8707.

Polat U, Sagi D (1993) Lateral interactions between spatial channels: suppression and facilitation revealed by lateral masking experiments. Vision Res 33:993-999.

Polat U, Sagi D (1994) The architecture of perceptual spatial interactions. Vision Res 34:73-78.
Polat U, Sagi D (2006) Temporal asymmetry of collinear lateral interactions. Vision Res 46:953-960.

Polat U, Sagi D (2007) The relationship between the subjective and objective aspects of visual filling-in. Vision Res 47:2473-2481.

Polat U, Tyler CW (1999) What pattern the eye sees best. Vision Res 39:887-895.

Polat U, Mizobe K, Pettet MW, Kasamatsu T, Norcia AM (1998) Collinear stimuli regulate visual responses depending on cell's contrast threshold. Nature 391:580-584.

Roberts M, Delicato LS, Herrero J, Gieselmann MA, Thiele A (2007) Attention alters spatial integration in macaque $\mathrm{V} 1$ in an eccentricity-dependent manner. Nat Neurosci 10:1483-1491.

Roelfsema PR, Lamme VA, Spekreijse H (1998) Object-based attention in the primary visual cortex of the macaque monkey. Nature 395:376-381.

Roelfsema PR, Khayat PS, Spekreijse H (2003) Subtask sequencing in the primary visual cortex. Proc Natl Acad Sci U S A 100:5467-5472.

Roelfsema PR, Tolboom M, Khayat PS (2007) Different processing phases for features, figures, and selective attention in the primary visual cortex. Neuron 56:785-792.

Sceniak MP, Ringach DL, Hawken MJ, Shapley R (1999) Contrast's effect on spatial summation by macaque V1 neurons. Nat Neurosci 2:733-739.

Seriès P, Lorenceau J, Frégnac Y (2003) The "silent" surround of V1 receptive fields: theory and experiments. J Physiol Paris 97:453-474.

Shani R, Sagi D (2005) Eccentricity effects on lateral interactions. Vision Res 45:2009-2024.

Supèr H, Roelfsema PR (2005) Chronic multiunit recordings in behaving animals: advantages and limitations. Prog Brain Res 147:263-282.

Thiele A, Distler C, Korbmacher H, Hoffmann KP (2004) Contribution of inhibitory mechanisms to direction selectivity and response normalization in macaque middle temporal area. Proc Natl Acad Sci U S A 101:9810-9815.

Thiele A, Delicato LS, Roberts MJ, Gieselmann MA (2006) A novel electrode-pipette design for simultaneous recording of extracellular spikes and iontophoretic drug application in awake behaving monkeys. J Neurosci Methods 158:207-211.

Watson AB (1979) Probability summation over time. Vision Res 19: $515-522$.

Wehrhahn C, Dresp B (1998) Detection facilitation by collinear stimuli in humans: dependence on strength and sign of contrast. Vision Res 38:423-428.

Williams CB, Hess RF (1998) Relationship between facilitation at threshold and suprathreshold contour integration. J Opt Soc Am A Opt Image Sci Vis 15:2046-2051.

Williford T, Maunsell JH (2006) Effects of spatial attention on contrast response functions in macaque area V4. J Neurophysiol 96:40-54.

Xing J, Heeger DJ (2000) Center-surround interactions in foveal and peripheral vision. Vision Res 40:3065-3072.

Zenger-Landolt B, Koch C (2001) Flanker effects in peripheral contrast discrimination—psychophysics and modeling. Vision Res 41:3663-3675. 